\title{
Género y Economía en Latinoamérica. Análisis Sistemático de la Producción Científica en Scopus
}

\author{
Claudia Patricia Maldonado-Erazo*, Pablo Quiñonez ${ }^{\dagger}$
}

\section{Resumen:}

En este artículo se realiza un estudio bibliométrico de carácter exploratorio sobre la situación actual y la evolución histórica de las investigaciones publicadas en revistas de alto impacto (indexadas en Scopus) sobre temas en que exista una intersección de las áreas de género y economía en América Latina. Los resultados muestran que, si bien desde la aparición de los estudios de género en la región estas dos áreas han estado estrechamente vinculadas, el número de productores importantes aún no es considerable, lo que podría estar relacionado con la juventud del tema en la región. Además, y en concordancia con las tendencias mundiales, se encontró que la mayoría de autores que trabajan en estas áreas son mujeres y se encuentran en los centros de conocimiento occidentales tradicionales, como los Estados Unidos y el Reino Unido. Por último, se observó que predominaban los investigadores transitorios en el campo y que alrededor del $50 \%$ de las investigaciones se habían publicado en revistas situadas en el cuartil de mayor impacto.

\section{Códigos JEL: B54, N36}

Éste es un preprint del capítulo titulado Gender and Economics in Latin America: a Systematic Analysis of Scientific Production in Scopus, a ser publicado en Quiñonez, P., \& Maldonado-Erazo, C. (Eds.) (2021). Gender Inequality in Latin America. The Case of Ecuador. Brill. https://doi.org/10.1163/9789004442917

\section{Introducción}

En el contexto de un sistema económico y social marcado por notorias desigualdades, las mujeres se encuentran y se han encontrado en una situación de desventaja a lo largo de la historia en muchos ámbitos, incluyendo al mundo de la academia. De hecho, desde una perspectiva histórica, la presencia de mujeres como estudiantes y profesoras en las universidades del mundo es un hecho relativamente reciente $y$, aunque su presencia ha crecido notoriamente en los últimos años, las mujeres aún se encuentran en desventaja en prácticamente todas las facetas del mundo académico, inclusive en los países desarrollados (Shen, 2013).

\footnotetext{
${ }^{*}$ C. P. Maldonado-Erazo $(\bowtie)$

Escuela Superior Politécnica de Chimborazo, Ecuador

E-mail: claudia.maldonado@espoch.edu.ec

$\dagger$ P. Quiñonez

Universidad de Guayaquil, Ecuador

E-mail: pablo.quinonezr@ug.edu.ec
} 
Por ejemplo, en la Unión Europea, pese a los constantes avances de los últimos años, apenas un tercio de las investigadoras son mujeres; éstas continúan subrepresentadas en la publicación de artículos científicos, en el registro de patentes y en la recepción de becas de investigación; en 2017 apenas el 22\% de las instituciones de educación superior eran lideradas por mujeres; y, a 2014, las mujeres en el área de investigación y desarrollo ganaban un 17\% menos, en promedio, que sus colegas hombres (European Commision, 2019).

Por supuesto, este sesgo desfavorable para las mujeres tiene su raíz en una matriz específica de pensamiento que tiende a auto reforzarse a sí misma y a los fenómenos derivados de ella y que, en la práctica, pone a prueba a la idea de meritocracia predominante en el mundo académico. Tal es la realidad que, por ejemplo, numerosos estudios han encontrado que inclusive desde la infancia el rol de un científico habitualmente es asociado con los hombres (Cvencek, et al., 2011; Steinke et al., 2007). Por todo lo mencionado, no es de extrañarse que inclusive después de considerar factores que pudiesen afectar el nivel de éxito en la carrera académica, exista una gran cantidad de evidencia que demuestra que los esfuerzos y los logros académicos de las mujeres son menos valorados y premiados que los de los hombres (Ellemers, 2018; Van der Lee \& Ellemers, 2018).

Como ya se insinuó anteriormente, en el campo específico de la publicación de artículos científicos, la realidad es similar. Un estudio reciente que consideró a más de 10 millones de artículos publicados desde 2002, cubriendo gran parte de las disciplinas de STEMM (Science, Technology, Engineering, Mathematics, and Medicine), encontró que, pese al reciente progreso, la brecha de género en el área aún está lejos de cerrarse y que, inclusive, en ciertas disciplinas como cirugía, ciencias informáticas, física y matemáticas, al ritmo actual, la brecha no se podría cerrar en el presente siglo (Holman, et al., 2018).

Y tal realidad, que se vuelve más notoria conforme mayor es el prestigio de las revistas académicas, no es exclusiva de las disciplinas de STEMM, aunque probablemente en otras áreas no sea tan aguda. Por ejemplo, en el campo de las ciencias sociales, Teele y Thelen (2017) encontraron, luego de revisar a 10 de las más prestigiosas revistas de Ciencia Política, que el bajo porcentaje de autoras que publican en ellas no reflejan simplemente un bajo porcentaje de mujeres en esa profesión; sino que, por el contrario, existe una subrepresentación de las mujeres en las revistas más prominentes de esa disciplina, sumado al hecho de que la mayoría de la investigación colaborativa en estas revistas proviene de equipos exclusivamente masculinos. Una realidad no distante de las demás disciplinas de estudio.

A ello se suma el hecho de que la investigación tendiente a visibilizar estos problemas habitualmente se ha visto marginada en el mundo académico. Por ejemplo, Cislak et al. (2018) encontraron evidencia, luego de una investigación bibliométrica en un amplio rango de ciencias sociales, de que los artículos relativos al sesgo de género recibían menor financiamiento y a menudo eran publicados en revistas con menor factor de impacto que otros artículos comparables sobre discriminación social. Esto refleja no solamente mecanismos explícitos de discriminación, sino también mecanismos más sutiles y relacionados con un sesgo negativo en la calidad percibida de los estudios sobre discriminación de género (Handley et al., 2015). 
Además de ello, los estudios de género habitualmente han sido encasillados y vinculados exclusivamente con el compromiso a la acción de militantes de dicha perspectiva. De hecho, existe abundante evidencia que apunta a que quienes realizan investigación en áreas relativas al género, en diferentes disciplinas, son mayoritariamente mujeres (Caro González \& Guarinos, 2017; Söderlund \& Madison, 2015).

Adicionalmente, es notorio que la mayoría de la investigación en torno a temas de género y relacionados proviene de los centros occidentales tradicionales del conocimiento, haciendo que el estar ubicado en una universidad norteamericana o de Europa occidental parezca ser un factor crucial para que los autores sean publicados y citados (Wöhrer, 2016). De hecho, el 79,5\% de la literatura sobre estudios de las mujeres, entre 1900 y 2013, ha provenido de Estados Unidos y el Reino Unido (Tsay \& Li, 2017).

En lo que refiere a América Latina, el interés académico desde las ciencias sociales por la situación de la mujer empieza a evidenciarse desde la década de 1970, sobre todo en temas relacionados al campo del desarrollo y los debates que en torno a este tema prevalecían en ese momento en la región y el mundo. Sin embargo, pese a la aparición de estos estudios pioneros, generalmente agrupados en la categoría de 'women's studies', no sería sino hasta mediados de la década de 1980 que surgiría una cantidad relativamente grande de estudios que usaban al género como una categoría de análisis (Caulfield, 2001). No es de extrañar, por tanto, que Ann Pescatello, a inicios de la década de 1970, al realizar un estudio bibliográfico sobre el tema, lamentase el subdesarrollo, en general, de los estudios sobre mujeres latinoamericanas, encontrando un campo tan incipiente que le fue difícil identificar las principales tendencias y autores (Pescatello, 1972).

La progresiva preferencia por el uso del concepto de género en lugar de la categoría de estudios sobre las mujeres, pese a los grandes puntos en común en lo referente a la agenda de investigación de ambos conceptos, obedece al carácter eminentemente biológico del segundo, en contraste con la naturaleza del primero como una construcción social que limita comportamientos, lugares y relaciones de poder entre lo masculino y lo femenino (Abbassi \& Lutjens, 2002). En ese sentido, el uso de 'género' refleja el esfuerzo por parte de los académicos para evitar cierto esencialismo, entendido como el tratamiento de los fenómenos sociales como externos y previos a las estructuras, prácticas y discursos sociales y culturales que les dieron origen, como explica Jackson (1998).

En este contexto, el presente capítulo pretende echar luz sobre la situación actual y la evolución histórica de la investigación publicada en revistas de alto impacto en el área de género y economía en América Latina, dos campos estrechamente vinculados en la investigación en torno a los asuntos de género desde su surgimiento mismo en la región. Para tal fin, se realizará un análisis bibliométrico, de carácter exploratorio y cuantitativo, contemplando la producción que se encuentra indexada dentro de la base de datos Scopus del grupo Elsevier y que centra su estudio de manera específica en temas relativos al género y la economía en la región.

Para ello, el presente capítulo se estructura de la siguiente manera: en la sección 2 se presenta una revisión de literatura sobre las discusiones de género y economía en la región, en la 
sección 3 se detalla la metodología empleada, en la sección 4 se detallan los resultados, en la sección 5 se presentan las conclusiones y en la sección 6 las limitaciones del presente estudio.

\section{La investigación sobre género y economía en América Latina}

Como conocemos, el termino género se ha usado tradicionalmente para referirse a las diferencias biológicas entre hombres y mujeres (Castaño, 1999), y luego paulatinamente ha pasado a ser considerado como una serie de construcciones dialécticas que enmarcan funciones y acciones puntuales a cada uno de ellos, que en muchos de los casos se originan desde la construcción social de la cultura de los distintos grupos humanos. Estas construcciones han mostrado una evidente separación de actividades, roles y actitudes, asociando a la mujer únicamente con actividades del hogar, con la sumisión, intuición o como el puente conexión con la naturaleza y/o los demás; en tanto que el hombre se ha visto ubicado en una instancia que podría considerarse inclusive relativamente superior, por considerárselo capaz de ejecutar relaciones con el mercado, el poder o la esfera pública.

De esta forma, alrededor del género se ha establecido un sistema de relaciones en el cual se han desarrollado espacios de dominio (visible, masculino) y dominación (invisible, femenino) (Blau et al., 2002; Carrasco, 2003; Vásconez, 2017). En lo que refiere al estudio de este fenómeno, es a partir de los años 70 y 80, especialmente, que se aprecia un interés considerable y creciente por su estudio desde las ciencias sociales y es durante este periodo que el concepto empieza a apreciarse como algo que va más allá de cuestiones culturales o biológicas, siendo más bien una categoría transdisciplinaria que toma forma propia, a partir de la cual se crean comprensiones de las actividades, creencias, valores y conductas que son socialmente apreciadas y diferentes para mujeres y hombres, y las cuales deben ser estudiadas (Benería \& Roldán, 1992).

Sin embargo, la lucha en contra de referida discriminación probablemente puede situarse mucho antes del auge en el interés académico por el tema, al menos en América Latina. En esta región, entre las primeras acciones que ponen en "igualdad de derechos" a la mujer con el hombre, se encuentra la conquista del derecho al sufragio, ocurrida por primera vez en 1924 con el voto de Matilde Hidalgo Navarro, en Ecuador. Pese a ello, se aprecia que, a pesar de poder ejercer dicho derecho, la participación política de la mujer latinoamericana sigue siendo restringida aún al día de hoy (Desposato \& Norrander, 2009).

Volviendo a la discusión sobre el surgimiento del interés académico en el área en América Latina, es alrededor de 1970 que se registran los primeros estudios sobre la desigualdad a la que se enfrenta la mujer y su influencia en el desarrollo de la región (Lovell, 2006). Ello encaja, de cierta manera, con una segunda ola del movimiento feminista a nivel internacional, que entre 1960 y 1970 dio lugar a la producción sistemática de conocimientos inexistentes hasta ese momento, pero que permitían una comprensión más amplia sobre las condiciones de las mujeres (De Barbieri, 2004). Sin embargo, es difícil profundizar en el estudio sobre las mujeres latinoamericanas durante estos primeros años, puesto que las primeras investigaciones no alcanzaron espacios de divulgación que las posicionen de la mejor manera en el mundo académico, además de apreciarse en los intelectuales poco entusiasmo por las 
causas feministas ${ }^{1}$ como en su momento lo diagnosticó Pescatello (1972). Esta autora, en un pionero estudio bibliográfico sobre el tema, identificó 70 diferentes documentos que para ese entonces ya abordaban temas de la realidad de la mujer latinoamericana.

De manera interesante, fue la investigación en el campo del desarrollo la que hizo que los estudiosos de las ciencias sociales en América Latina pusiesen por primera vez sus ojos en temas relacionados con la mujer (Navarro, 1979). Por aquel entonces, empieza a sacarse a la luz la sesgada participación laboral, la centralización de roles productivos y los desiguales niveles de desarrollo que la mujer enfrenta en diferentes espacios (Nash \& Safa, 1976; Saffioti, 1969).

Algo interesante durante la década de los 70s es que, aunque varios académicos en el área de las ciencias sociales en América Latina trabajaron en estudios de la mujer desde la perspectiva marxista (algo relativamente común a nivel global en aquel momento), la mayoría siguió el enfoque de la teoría de la dependencia, ampliamente popular en la región en ese entonces (Navarro, 1979). Destacan trabajos como los de Jelin (1974), Madeira (1978), Blay (1978) y Machado Neto (1978) para el caso de Brasil; de Lattes et al., (1977) para Argentina, Bolivia y Paraguay; Schmukler (1978) para Argentina; León de Leal (1977) para Colombia; Lustig et al., (1979) para México, entre otros.

Con la llegada de los 80s la investigación en el área crecería considerablemente, proyectándose con solidez hacia los $90 \mathrm{~s}$, y empleándose con creciente frecuencia el término género, algo muy poco frecuente en los trabajos publicados en décadas anteriores. En esta época, se exploran a profundidad temas relativos a la participación productiva de la mujer y sus contribuciones al desarrollo económico pese a la marginación a la que frecuentemente se veían enfrentadas (Abramo, 1998; Brockman, 1985; Cabral, 1994; Hays-Mitchell, 1997; Kidder, 1999; Morales i Perez, 1999; Pacheco \& Blanco, 1998; Rowlands, 1992), división sexual del trabajo e inequidades en las condiciones laborales y salariales (Laurie, 1997, 1999; Radcliffe, 1990; Raynolds, 1998; Roberts \& Dodoo, 1995), y participación en las normas de organización política local, nacional o internacional (Hays-Mitchell, 1995; Moreno, 1996; Rico, 1998; Teissedre, 1997), además de una amplia variedad de estudios en torno a impactos socio-económicos (Aslanbeigui et al., 1994; Balan, 1990; Chant, 1991; Cravey, 1997; Guendelman, 1987; Molinas, 1998; Psacharopoulos \& Tzannatos, 1993; Scott, 1986).

Entrado el siglo XXI, la investigación en torno al tema continuaría profundizándose y expandiéndose (ver, por ejemplo, Deere \& León, 2000; Salvador, 2007; Esquivel, 2011; Reyes, 2011; Rodríguez Enríquez, 2015, entre otros). Con ello, los estudios en torno a las posibilidades y alternativas de desarrollo para países del Sur global, como los de América Latina, se han visto enriquecidos con la incorporación del género como una variable de análisis (Rodríguez Enríquez, 2007), aunque no haya logrado los niveles de difusión que en el mundo desarrollado.

\footnotetext{
${ }^{1}$ En América Latina los estudios sobre la mujer no surgieron de un vigoroso movimiento de liberación femenina (como sí sucedió en EE. UU. y en algunos países de Europa); y, de hecho, de entre los académicos pioneros en estudios en esta disciplina en la región, varios no se consideraban feministas (Navarro, 1979).
} 


\section{Metodología}

La metodología empleada se estructura en relación a la aplicación de un análisis bibliométrico de carácter exploratorio y cuantitativo, enfocado en la producción científica identificada en relación a género y economía en Latinoamérica, contemplando la producción que se encuentra indexada dentro de la base de datos internacional Scopus del grupo Elsevier.

La selección de esta base de datos está relacionada con el hecho de que ésta contempla una mayor cobertura en tiempo, elemento que influye en los datos de citación de las unidades documentales, debido a que los índices de citas/unidad documental global son superiores en Scopus (Hernández-González et al., 2016).

Adicional a ello, esta base de datos admite una mayor descarga (máximo 2000 referencias) de metadatos que permiten obtener información bibliográfica, resumen, palabras clave, detalles por autor, etc. (Fernández et al., 1999). Finalmente, esta base dispone de rigurosos estándares de calidad como el presente en SJR (Scimago Journal Rank) que transparenta la calidad de la información suministrada por la base (Harzing \& Alakangas, 2016).

Se establece como unidad documental de análisis el artículo, debido a que este tipo de documento se caracteriza por permitir un rápido acceso a la literatura científica (Frank, 2006), además de albergar las contribuciones científicas más recientes y de gran valor para la comunidad científica (Goldschmidt, 1986). El proceso empleado para la consolidación de la matriz de unidades documentales de presenta en la Figura 1.

En este tipo de análisis se plantea el desarrollo de una "bibliometría", proceso que, apoyado en la estadística, permite el tratamiento e interpretación de diferentes metadatos recolectados dentro de la base de indexación seleccionada. Cabe mencionar que, los metadatos se han establecido en la última década como la principal fuente de información para muchos estudios científicos (Durán-Sánchez et al., 2018), convirtiéndose de esta manera en los principales datos para la aplicación de diversos tipos de indicadores bibliométricos (Spinak, 1996), los cuales surgen de varios modelos matemáticos que se fundamentan en la relación de dos o más variables (Hubert, 1981).

De acuerdo con Escorcia-Otálora y Poutou-Piñales (2008) los indicadores se pueden agrupar en dos grandes grupos. El primero agrupa al conjunto de indicadores de actividad, es decir, son aquellos que se emplean para conocer el estado actual del área del conocimiento; mientras que, el segundo recoge indicadores de impacto que determinan la relevancia de la información a nivel internacional.

Los indicadores bibliométricos implican la generación de un amplio listado del cual se pueden crear un número infinito de combinaciones, por lo que termina siendo esencialmente inadmisible el poder aseverar que se puede establecer una combinación perfecta para el desarrollo de este tipo de estudios. Más allá de ello, deben considerarse los argumentos de autores como Bonilla et al., (2015), quienes afirman que la calidad y relevancia del análisis efectuado dentro de los estudios bibliométricos se encuentra en una relación directamente proporcional con la combinación de indicadores aplicados para la evaluación de los metadatos de cada unidad documental seleccionada. 


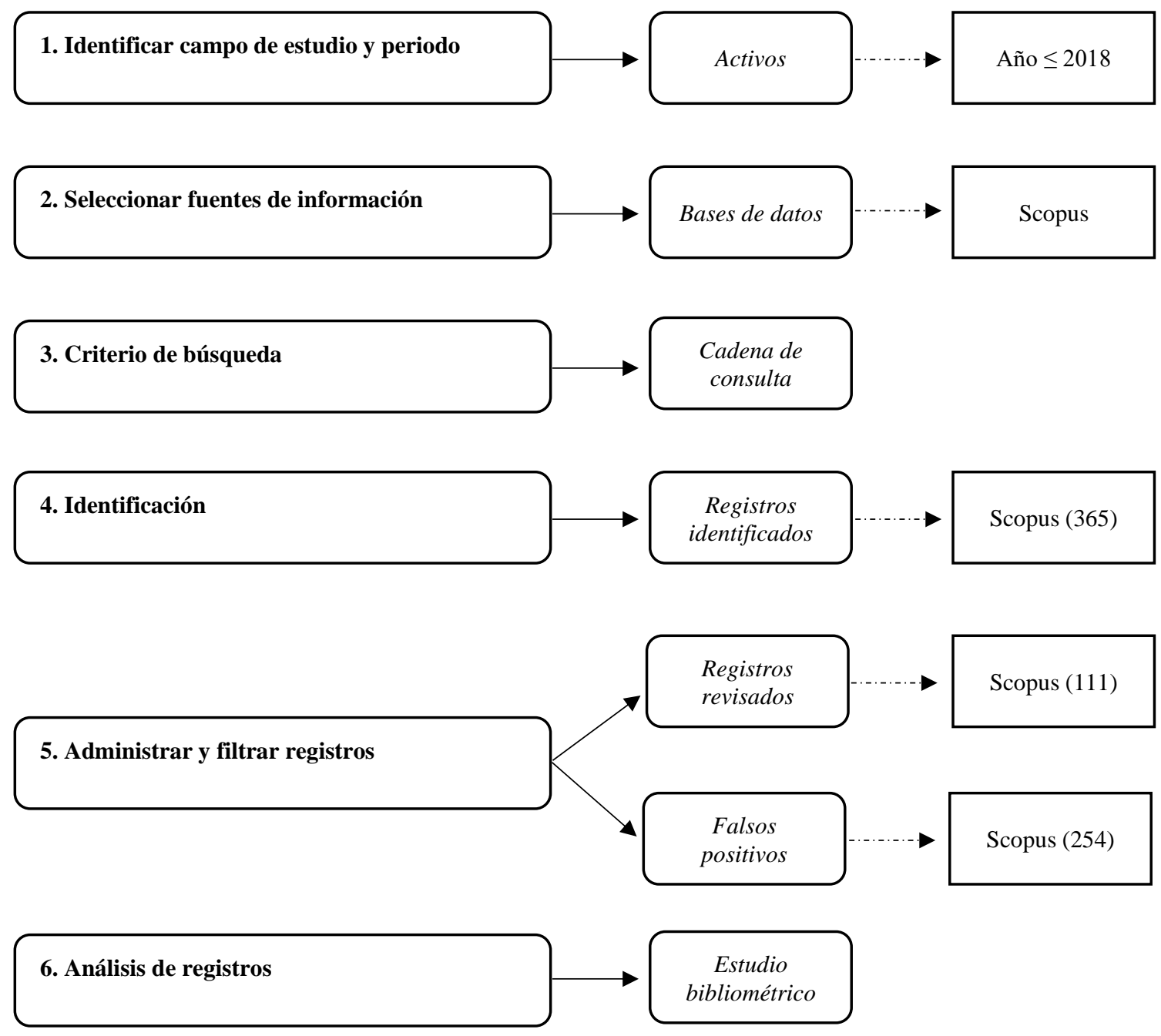

Figura 1. Procedimiento metodológico bibliométrico

Nota: Elaboración propia

En complemento, es necesario mencionar que no solo la cantidad de indicadores es determinante para establecer la calidad de estudio, sino que también es necesario considerar que esta deberá ser evaluada en función del enfoque que se pretende dar al estudio; para ello Alonso et al., (2009) determinan que la elección y combinación de indicadores debe efectuarse en función de la adaptabilidad que tengan estos para los propósitos a ser conseguidos.

En resumen, la elaboración de una bibliometría se establece como la consolidación de una herramienta efectiva para la organización de la información académica existente hasta el momento, puesto que suministra información muy detallada y útil para los investigadores en función de la rama analizada, debido a que por un lado, rastrea la mayor cantidad de material bibliográfico con injerencia directa para el tema (Álvarez-García et al., 2018; Andrés, 2009); mientras que por otro, determina el comportamiento productivo existente dentro de dicha rama (Podsakoff et al., 2008).

Tomando en consideración todo lo antes indicado, los indicadores bibliométricos a ser 
empleados se centran en: evolución de las publicaciones, autores más prolíferos, países más productores, tipo de instituciones centradas en la investigación de dicha temática, citación de artículos, revistas con mayor concentración de artículos, relaciones de co-citación y coautorías. Además de introducir dentro de todos los indicadores un análisis complementario de género que permitirá establecer la participación de hombres y mujeres dentro del desarrollo de este tipo de estudios.

El rastreo de las unidades documentales se desarrolla por medio de búsquedas avanzadas de datos, sustentadas en los criterios y ecuación de búsqueda descritos en la tabla 1. El uso de dichos criterios permite integrar dentro de los resultados revistas provenientes de la mayor cantidad de áreas de conocimiento, con lo cual las búsquedas se convierten en un proceso mucho más exhaustivo y de calidad (Álvarez-García et al., 2018; Corral \& Cànoves, 2013).

Tabla 1. Estrategia de búsqueda

\begin{tabular}{ll}
\hline Términos de búsqueda & gender, econom*, Latin America \\
\hline Título de la categoría & Título del artículo, resumen, palabras claves \\
\hline Área temática & Todas \\
\hline Tipo de documento & Artículo \\
\hline Periodo de tiempo & Año de publicación $\leq 2018$ \\
\hline Lenguaje & Todos \\
\hline Ecuación de búsqueda & ( TITLE-ABS-KEY ( "gender" ) AND TITLE-ABS-KEY ( "econom*" ) AND \\
\hline Fecha de búsqueda & TITLE-ABS-KEY ( "Latin America" )) AND DOCTYPE ( ar ) \\
\hline
\end{tabular}

Nota: Elaboración propia

Una vez consolidado el rastreo, se procedió a la gestión y selección de las unidades documentales, proceso durante el cual se refinaron los datos recopilados al eliminar documentos duplicados o aquellos carentes de relación a la temática de estudio, esta última situación generada por el inadecuado uso de palabras claves por parte de los autores durante la indexación de los mismos. Seguido, se elaboró de una base de datos ad hoc en función de las variables de análisis requeridas para la aplicación de cada indicador bibliométrico seleccionado, considerando un total de 111 artículos.

\section{Resultados}

\section{Productividad por años}

La trayectoria de estudio se encuentra concentrada en Scopus a lo largo de 34 años, dentro de los cuales se ha consolidado un total de 111 artículos. El primer estudio indexado en relación a género y economía en Latinoamérica es Women and development in northern Belize trabajo desarrollado por Brockman en el año 1985. 


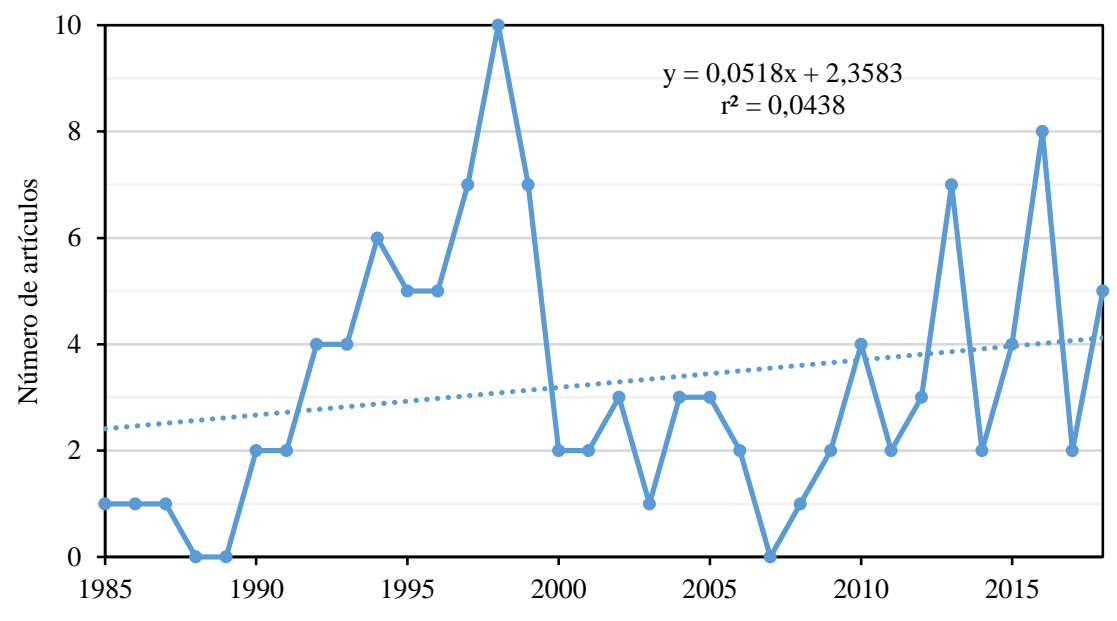

Gráfico 1. Evolución de la publicaciones en Scopus

Nota: Elaboración propia

El gráfico 1 presenta una línea de evolución entre los años 1985 al 2018, periodo en el cual 1998 se consolida como el año de mayor productividad con 10 artículos publicados. Dicho año se establece como un referente de cambios dentro de Latinoamérica al suscitarse varios eventos; en el caso de Perú la Defensoría del Pueblo expresa la importancia del respeto de los derechos reproductivos reconocidos en el Art. 6 de la Constitución de dicho país en respuesta a las acciones ejecutadas para regular el número de hijos que una mujer podía tener; en Argentina en la Constitución de Salta se incorpora la no discriminación por sexo, se promueve la igualdad jurídica en varios planos, así como impulsar el no sexismo en lenguaje de cargos públicos; así también en Ecuador se consigue la integración de 35 reformas constitucionales que permiten la incorporación de los derechos de las mujeres dentro de la Constitución del 1998, acto que al mismo tiempo resalta la vulnerabilidad jurídica existente en otros territorios, en los cuales derechos elementales (voto, divorcio, aborto, participación pública, estudios, entre otros) continuaban siendo excluidos para las mujeres (Montaño \& Aranda, 2006).

En relación al comportamiento de la línea de evolución, esta se analiza en función de las fases de comportamiento establecidas por López López (1996), quien menciona la existencia de tres fases, dentro de las cuales cada una determina el estado y avance alcanzado dentro del estudio de cualquier área de conocimiento. Las fases que se pueden identificar son: precursores, crecimiento exponencial y crecimiento lineal.

Dentro de la línea de evolución de la presente temática, se identifica la presencia de dos fases: la primera, denominada como fase de precursores, se desarrolla de 1985 a 1991, y en ella se aprecia una relación de 1,00 artículos/año, y en los cuales un 62,5\% de las autoras son mujeres. La presencia de una incipiente producción de artículos está relacionada a la reducida participación de autores en este periodo de tiempo, establecido como el de nacimiento para el estudio de la temática. La segunda fase o fase de crecimiento exponencial, se presenta de 1992 a 2018 con una relación de 3,85 artículos/año. En este periodo se aprecia un aumento sustancial en el estudio de la temática, además de evidenciarse un aumento en el total de autores masculinos (48, frente a 3 de la fase de precursores), que, sin embargo, ocurre a un 
ritmo menor al del crecimiento de la participación femenina, originando que en la segunda fase que corresponde a que $70,4 \%$ de los autores sean mujeres.

Aunque no se aprecia una tendencia continua de crecimiento en la producción académica registrada en Scopus, es innegable que la literatura en relación a género y economía es notablemente superior en la fase de crecimiento exponencial que en la fase de precursores, hecho que concuerda ciertamente con la Ley de Price, la cual menciona que luego de 10 a 15 años de haber iniciado el estudio de cualquier temática, la información desarrollada sobre el tema a nivel global se verá duplicada (Price, 1956).

Pese a ello, se aprecia que la relación entre la producción y el tiempo transcurrido dentro de esta temática no se consolida como una condición determinante que permita predecir con certeza el comportamiento de evolución que presentará la temática, siendo necesario contrastar esta con otros indicadores.

\subsection{Citación}

Se contabiliza que en los 34 años de evolución se ha realizado un total de 1330 citas en relación a los 111 artículos de estudio, con una relación de 11,98 citas/artículos. El comportamiento de citado de estos documentos se expresa en un índice $\mathrm{h}=20$, con lo cual al menos 20 artículos del total de documentos identificados han obtenido 20 citas o más, hecho que se aprecia dentro del gráfico 2. El año que alcanza el mayor número de citas es 2002 con 258 citas, concentrando el 19,4\% del total de citas de la base.

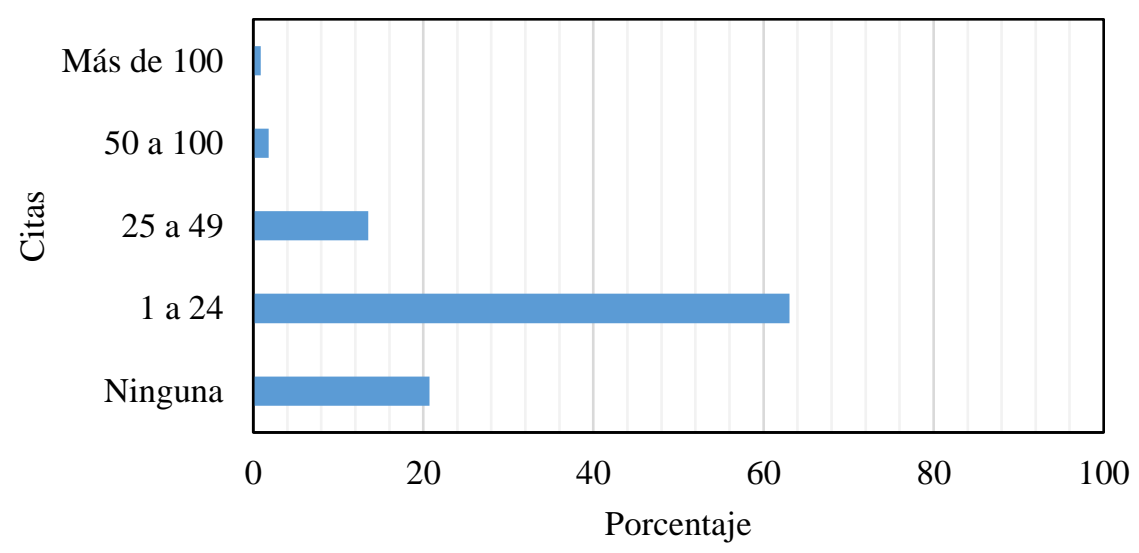

Gráfico 2. Distribución de las citas en Scopus

Nota: Elaboración propia

Del total general de artículos, el $63 \%$ ha obtenido entre 24 a 1 cita, mientras que el $21 \%$ de los artículos no registran citas durante el periodo de análisis. Por otro lado, tan solo el $14 \%$ (15) de los trabajos obtienen entre 25 a 100 citas y tan solo el $1 \%$ de los artículos registran más de 100 citas durante todo el periodo de análisis.

En cuanto al comportamiento en el citado de los artículos, se aprecia que los documentos 
publicados en los últimos 10 años carecen de un número considerable de citas debido a que dichos estudios no han conseguido la divulgación necesaria para consolidarse como referentes de la temática, hecho que limita la cantidad de citas que estos pueden percibir (Merigó et al., 2015).

Tabla 2. Ranking de los articulos más citados

\begin{tabular}{|c|c|c|c|c|c|}
\hline$R$ & Autores & Título & 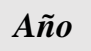 & Citas & $C / A$ \\
\hline 1 & Molyneux & $\begin{array}{l}\text { Gender and the silences of social capital: } \\
\text { Lessons from Latin America }\end{array}$ & 2002 & 231 & 13,6 \\
\hline 2 & Molinas & $\begin{array}{l}\text { The impact of inequality, gender, external } \\
\text { assistance and social capital on local-level } \\
\text { cooperation }\end{array}$ & 1998 & 82 & 3,9 \\
\hline 3 & $\begin{array}{l}\text { Desposato \& } \\
\text { Norrander }\end{array}$ & $\begin{array}{l}\text { The Gender Gap in Latin America: Contextual } \\
\text { and individual influences on gender and } \\
\text { political participation }\end{array}$ & 2009 & 52 & 5,2 \\
\hline 4 & $\begin{array}{l}\text { Aslanbeigui, } \\
\text { Pressman, \& } \\
\text { Summerfield }\end{array}$ & $\begin{array}{l}\text { Women in the age of economic transformation: } \\
\text { gender impact of reforms in post-socialist and } \\
\text { developing countries }\end{array}$ & 1994 & 41 & 1,7 \\
\hline 5 & Fuwa & $\begin{array}{l}\text { The poverty and heterogeneity among female- } \\
\text { headed households revisited: The case of } \\
\text { Panama }\end{array}$ & 2000 & 40 & 2,1 \\
\hline 6 & $\begin{array}{l}\text { Ellis, Conway, \& } \\
\text { Bailey }\end{array}$ & $\begin{array}{l}\text { The circular migration of Puerto Rican women: } \\
\text { Towards a gendered explanation }\end{array}$ & 1996 & 39 & 1,7 \\
\hline 7 & Lovell & $\begin{array}{l}\text { Race, gender, and work in São Paulo, Brazil, } \\
1960-2000\end{array}$ & 2006 & 38 & 2,9 \\
\hline 8 & Terjesen \& Amorós & $\begin{array}{l}\text { Female entrepreneurship in Latin America and } \\
\text { the Caribbean: Characteristics, drivers and } \\
\text { relationship to economic development }\end{array}$ & 2010 & 36 & 4,0 \\
\hline 9 & Caulfield & $\begin{array}{l}\text { The history of gender in the historiography of } \\
\text { Latin America. }\end{array}$ & 2001 & 36 & 2,0 \\
\hline 10 & Cravey & $\begin{array}{l}\text { The politics of reproduction: Households in the } \\
\text { Mexican industrial transition }\end{array}$ & 1997 & 34 & 1,5 \\
\hline 11 & Morgan \& Buice & $\begin{array}{l}\text { Latin American attitudes toward women in } \\
\text { politics: The influence of elite cues, female } \\
\text { advancement, and individual characteristics }\end{array}$ & 2013 & 30 & 5,0 \\
\hline 12 & Raynolds & $\begin{array}{l}\text { Harnessing women's work: restructuring } \\
\text { agricultural and industrial labor forces in the } \\
\text { Dominican Republic }\end{array}$ & 1998 & 30 & 1,4 \\
\hline 13 & Hays-Mitchell & $\begin{array}{l}\text { Voices and visions from the streets: gender } \\
\text { interests and political participation among } \\
\text { women informal traders in Latin America }\end{array}$ & 1995 & 30 & 1,3 \\
\hline 14 & Georges & $\begin{array}{l}\text { Gender, Class, and Migration in the Dominican } \\
\text { Republic: Women's Experiences in a } \\
\text { Transnational Community. }\end{array}$ & 1992 & 30 & 0,8 \\
\hline 15 & Stephen & $\begin{array}{l}\text { Women's weaving cooperatives in Oaxaca: An } \\
\text { indigenous response to neoliberalism }\end{array}$ & 2005 & 28 & 2,0 \\
\hline 16 & Hays-Mitchell & $\begin{array}{l}\text { Resisting austerity: A gendered perspective on } \\
\text { neo-liberal restructuring in Peru }\end{array}$ & 2002 & 26 & 1,5 \\
\hline
\end{tabular}




\begin{tabular}{|c|l|l|c|c|c|}
\hline $\boldsymbol{R}$ & \multicolumn{1}{|c|}{ Autores } & \multicolumn{1}{|c|}{ Título } & Añ & Citas & C/A \\
\hline 17 & Howes \& Singh & $\begin{array}{l}\text { Long-term trends in the world economy: the } \\
\text { gender dimension }\end{array}$ & 1995 & 26 & 1,1 \\
\hline 18 & Hite \& Viterna & $\begin{array}{l}\text { Gendering class in Latin America: How women } \\
\text { effect and experience change in the class } \\
\text { structure }\end{array}$ & 2005 & 25 & 1,8 \\
\hline 19 & Wiig & $\begin{array}{l}\text { Joint titling in rural Peru: Impact on women's } \\
\text { participation in household decision-making }\end{array}$ & 2013 & 24 & 4,0 \\
\hline
\end{tabular}

* $\mathrm{R}=$ Ranking; $\mathrm{C} / \mathrm{A}=$ promedio de citas recibidas por artículo al año.

Nota: Elaboración propia

Como se muestra en la tabla 2, los artículos más citados son: Gender and the silences of social capital: Lessons from Latin America de Molyneux (2002); The impact of inequality, gender, external assistance and social capital on local-level cooperation de Molinas (1998); y The Gender Gap in Latin America: Contextual and individual influences on gender and political participation de Desposato y Norrander (2009) cada uno con 231, 82 y 52 citas respectivamente. Dichos documentos presentan una participación equitativa dentro de su elaboración, es decir se registra la participación de 2 mujeres y 2 hombres entre los autores que producen los tres documentos.

A pesar de ser los artículos anteriores los documentos que acumulan la mayor cantidad de citas, son los trabajos Molyneux (2002), Desposato y Norrander (2009), y, Morgan y Buice (2013) los que se establecen como los de mayor promedio de citas/años.

\subsection{Autores}

En relación a los autores, se ha registrado la participación de 169 autores dentro de los 111 documentos de análisis, con lo cual se establece un índice de productividad por autor de 1,04 artículos.

La productividad de los autores se puede analizar por medio de distintos tipos de clasificación que contribuyen en el proceso de determinar el papel desarrollado por cada autor dentro de la temática de estudio, para el cumplimiento de dicho propósito en este estudio se utilizará la clasificación de Crane (1977), en la cual la producción por autores se puede explicar a través de cuatro grupos de autores: (1) grandes productores, aquellos que poseen una producción superior a 10 artículos; (2) productores moderados, autores que han producido entre 5 y 9 documentos; (3) aspirantes, comprende a autores que disponen entre 2 y 4 estudios, y (4) transeúntes, conformado por los autores que solo han producido un trabajo. Esta clasificación se plantea como una opción mucho más detallada al cálculo del Índice de Lotka (1926).

Tabla 3. Clasificación de los autores sistema Crane

\begin{tabular}{|c|c|c|c|c|c|}
\hline $\begin{array}{c}\text { Nro. de } \\
\text { artículos } \\
\text { por autor }\end{array}$ & $\begin{array}{c}\text { Nro. de } \\
\text { autores }\end{array}$ & $\%$ & IP & $\begin{array}{c}\text { Clasificación } \\
\text { de Crane }\end{array}$ & Autores \\
\hline 1 & 163 & 96,4 & 0,000 & Transeúntes & Otros autores \\
\hline
\end{tabular}




\begin{tabular}{|c|c|c|c|c|l|}
\hline 2 & 5 & 3,0 & 0,301 & \multirow{2}{*}{ Aspirantes } & $\begin{array}{l}\text { Radcliffe, S. A.; Espino, A.; } \\
\text { Laurie, N.; Lawson, V. A.; } \\
\text { Brown, L. A. }\end{array}$ \\
\cline { 3 - 4 } & 1 & 0,6 & 0,477 & & Hays-Mitchell, M. \\
\hline Total & 169 & 100,00 & \multicolumn{3}{|l}{} \\
\hline
\end{tabular}

* IP = Índice de Productividad; \% = Frecuencia relativa.

Nota: Elaboración propia

Los datos presentados dentro de la tabla 3 evidencian la existencia de dos grupos de autores en el estudio de esta temática. Por un lado, autores transeúntes con el 96,4\% del total de autores registrados (este tipo de autor se caracteriza por aportar únicamente con un documento dentro de la línea de evolución de la temática); mientras que, por otro lado, se identifican autores aspirantes con el 3,0\%, los cuales disponen de una mayor participación, pero esta sigue siendo de carácter reducido.

El índice de transitoriedad se ubica en 96,4\%, mostrando que los autores relacionados al estudio de género y economía en Latinoamérica son esporádicos, puesto que en su gran mayoría solo surgen una vez dentro de la temática, para luego de ello no continuar realizando aportaciones en el resto de la línea de evolución.

Tabla 4. Ranking de los autores más productivos

\begin{tabular}{|l|l|l|l|l|l|c|c|}
\hline $\mathbf{R}$ & \multicolumn{1}{|c|}{ Autores } & \multicolumn{1}{|c|}{ País } & \multicolumn{1}{|c|}{ Universidad } & f & TC & C/f & $\begin{array}{c}\text { h- } \\
\text { Index }\end{array}$ \\
\hline 1 & $\begin{array}{l}\text { Hays- } \\
\text { Mitchell, } \\
\text { M. }\end{array}$ & $\begin{array}{l}\text { United } \\
\text { States }\end{array}$ & Colgate University & 3 & 151 & 0,02 & 6 \\
\hline 2 & $\begin{array}{l}\text { Lawson, V. } \\
\text { A. }\end{array}$ & $\begin{array}{l}\text { United } \\
\text { States }\end{array}$ & Ohio State University & 2 & 2083 & 0,00 & 24 \\
\hline 3 & $\begin{array}{l}\text { Radcliffe, } \\
\text { S. A. }\end{array}$ & $\begin{array}{l}\text { United } \\
\text { Kingdom }\end{array}$ & University of Cambridge & 2 & 1568 & 0,00 & 24 \\
\hline 4 & $\begin{array}{l}\text { Brown, L. } \\
\text { A. }\end{array}$ & $\begin{array}{l}\text { United } \\
\text { States }\end{array}$ & Ohio State University & 2 & 1201 & 0,00 & 19 \\
\hline 5 & Laurie, N. & $\begin{array}{l}\text { United } \\
\text { Kingdom }\end{array}$ & University of Newcastle Upon Tyne & 2 & 1056 & 0,00 & 18 \\
\hline 6 & Espino, A. & Uruguay & University of the Republic of Uruguay & 2 & 3 & 0,67 & 1 \\
\hline
\end{tabular}

$* \mathrm{R}=$ Ranking; $\mathrm{f}=$ Número de artículos publicados; $\mathrm{TC}=$ Total de citas recibidas por artículos publicados; $\mathrm{C} / \mathrm{f}=$ Promedio de citas recibidas por artículos publicados; h-index = Índice de Hirsch.

Nota: Elaboración propia

El ranking de los autores más productivos (que se puede apreciar en la tabla 4) se consolida con el total de autores aspirantes, puesto que éstos han desarrollo más de una publicación dentro del área. El autor más productivo es Hays-Mitchell, M. con un total de 3 artículos y un Índice $\mathrm{h}=6$; seguido se ubica Lawson, V. A. con un $\mathrm{h}=24$ y 2 artículos; mientras que en tercer lugar se coloca Radcliffe, S. A. con un $\mathrm{h}=24$ y 2 trabajos en su haber. Adicional a ello, 
se identifica que el $100 \%$ de los autores más productivos que aparecen en el ranking en el área son mujeres, algo concordante con la tendencia descrita por autores como Söderlund \& Madison (2015) y Caro González \& Guarinos (2017) quienes resaltan la mayoritaria presencia de mujeres investigando temas relativos al género para diferentes áreas del conocimiento.

En lo referente a las tendencias de colaboración, Berelson (1952) determina que entre más variada y mayor es la colaboración registrada dentro de la elaboración de los documentos, mayor es la madurez de estudio que posee la temática (López López, 1996). En función de ello, dentro de esta temática se identifica que el 68,5\% (76) de los artículos, se han desarrollado de forma individual, dejando al 31,5\% (35) restante de los documentos bajo una producción de colaboración múltiple.

Dentro de la cuota de documentos producidos en colaboración múltiple, se evidencia que el $18,9 \%$ (21) se ha elaborado entre dos autores, seguido con el 9\% (10) de trabajos efectuados con tres autores y tan solo el 3,6\% (4) se han desarrollo entre cuatro o más autores, tal como detalla la gráfica 3 .

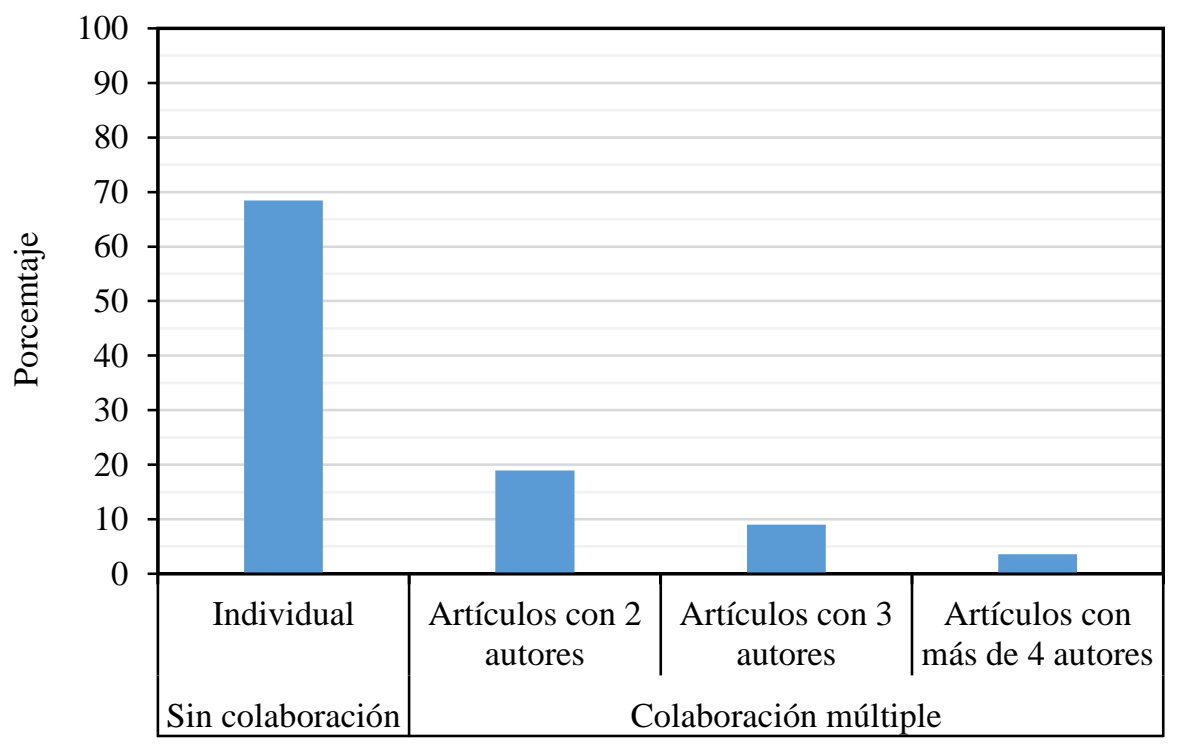

Gráfico 3. Coautorías

Nota: Elaboración propia

En complemento, el promedio general de colaboración que establece el índice de coautorías refleja una participación de 1,59 autores por artículo. El gráfico 4 detalla las relaciones de colaboración predominantes por tendencia de colaboración y en función del género, de esta manera los documentos producidos en pares, que es la tendencia de colaboración predominante, detallan que el $52,4 \%$ que se efectúan bajo la participación únicamente de mujeres, seguido del $28,6 \%$ de los documentos que se elaboran en colaboración mixta (participan tanto hombre como mujeres), en tanto que el 19\% presenta la colaboración solo de hombres. 


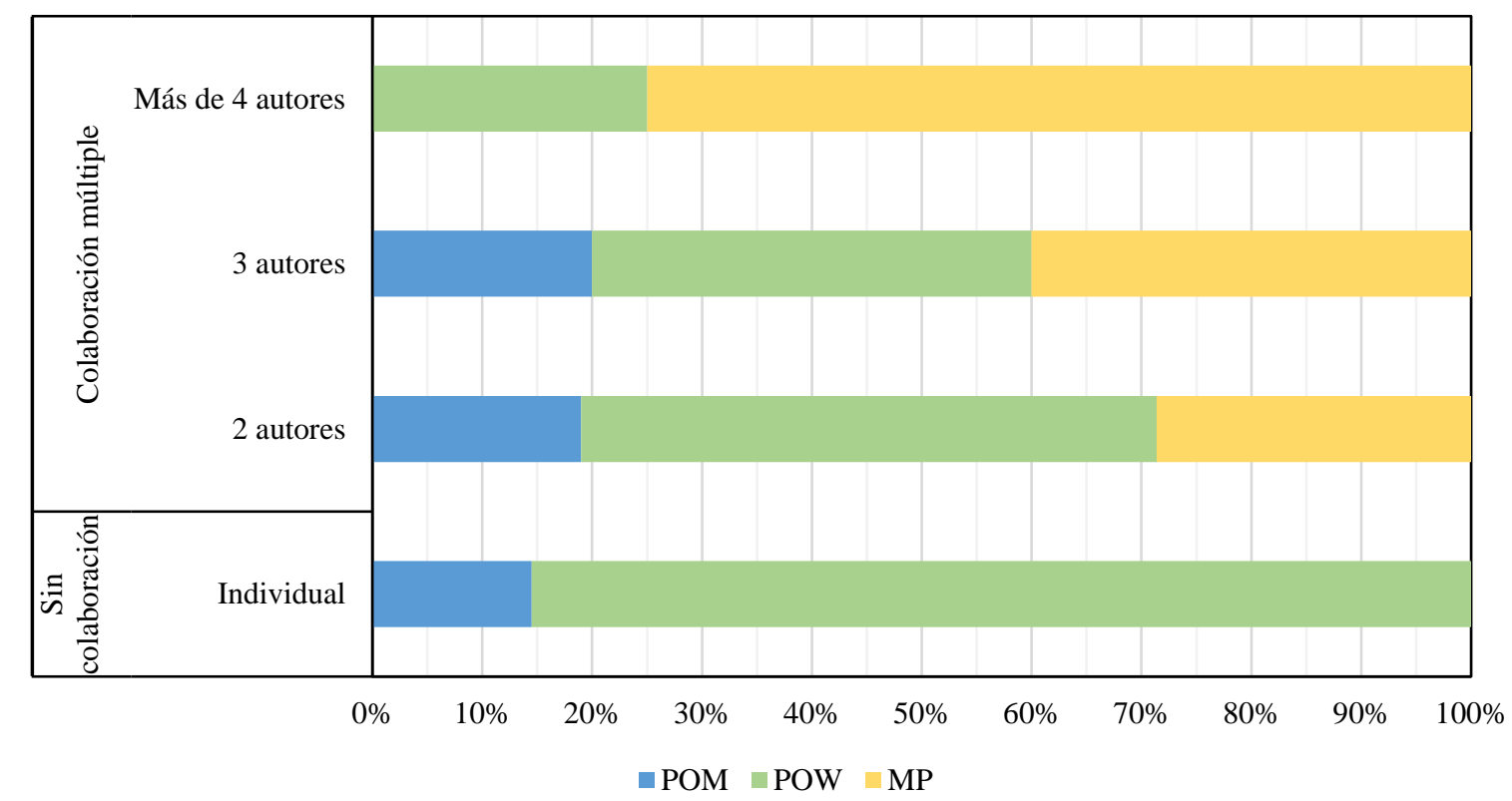

* POM = Participación solo de hombres; POW = Participación solo de mujeres; MP = Participación mixta.

Gráfico 4. Relaciones de co-autorías en función del género

Nota: Elaboración propia

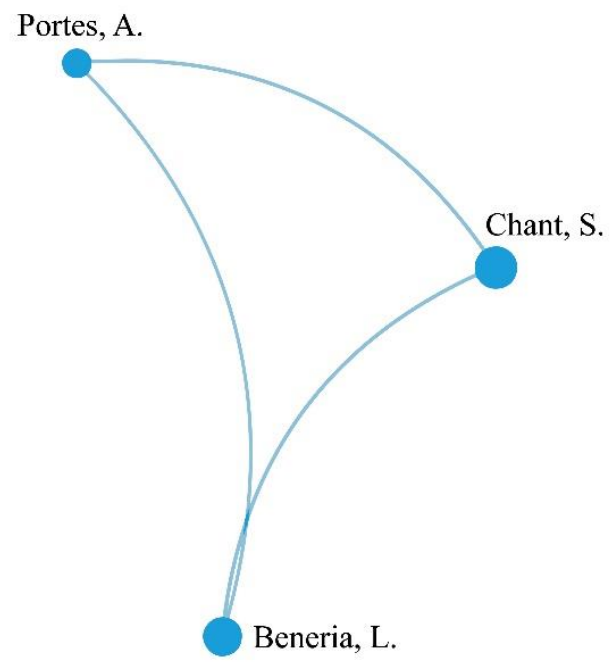

Gráfico 5. Co-citación de autores

Nota: Elaboración propia

Adicional a ello, se complementó el análisis de co-citación de autores, identificándose para ello los autores más citados entre sí. Dentro de esta temática, se aprecia la presencia de tres autores con mayor frecuencia de citado los cuales se detallan en la gráfica 5. El autor de mayor co-citación es Chant, S. con una frecuencia de 28 citas dentro del base de análisis. 


\subsection{Productividad por país e institución}

La productividad en función de la afiliación que registran los autores permite evaluar y comprender los nodos de información que se están desarrollando en función de las afiliaciones geográficas como institucionales registradas durante el periodo de estudio.

Por medio de un ranking de posiciones, se determina que por afiliación geográfica Estados Unidos es el principal nodo de producción al registrar 72 autores, 76 autorías y 50 centros, seguido de Chile con 13 autores, 13 autorías y 12 centros.

Tabla 5. Número de centros, autores y autorías por país de afiliación

\begin{tabular}{|c|l|c|c|c|}
\hline $\mathbf{R}$ & \multicolumn{1}{|c|}{ País } & A & As & C \\
\hline 1 & Estados Unidos & 72 & 76 & 50 \\
\hline 2 & Chile & 13 & 13 & 12 \\
\hline 3 & Reino Unido & 13 & 15 & 11 \\
\hline 4 & Argentina & 4 & 4 & 7 \\
\hline 5 & México & 8 & 8 & 6 \\
\hline 6 & Brasil & 5 & 5 & 5 \\
\hline 7 & España & 6 & 6 & 5 \\
\hline 8 & Perú & 4 & 4 & 4 \\
\hline 9 & Australia & 5 & 5 & 3 \\
\hline 10 & Canadá & 3 & 3 & 3 \\
\hline 11 & Colombia & 4 & 4 & 3 \\
\hline 12 & Ecuador & 4 & 4 & 3 \\
\hline 13 & Francia & 2 & 2 & 2 \\
\hline 14 & Países Bajos & 3 & 4 & 2 \\
\hline 15 & Uruguay & 1 & 1 & 1 \\
\hline 16 & Austria & 1 & 1 & 1 \\
\hline 17 & Bolivia & 2 & 2 & 1 \\
\hline 18 & Costa Rica & 1 & 1 & 1 \\
\hline 19 & Alemania & 1 & 1 & 1 \\
\hline 20 & Japón & 1 & 1 & 1 \\
\hline 21 & Nueva Zelanda & 1 & 1 & 1 \\
\hline 22 & Nicaragua & 8 & 8 & -- \\
\hline 23 & Noruega & & & \\
\hline 24 & Paraguay & Sin afiliación & 3 & 1 \\
\hline 25 & Autores; As $=$ Autorías; C $=$ Centros & & 1 & 1 \\
\hline Ranking: & & 2 & 2 \\
\hline
\end{tabular}

Nota: Elaboración propia 


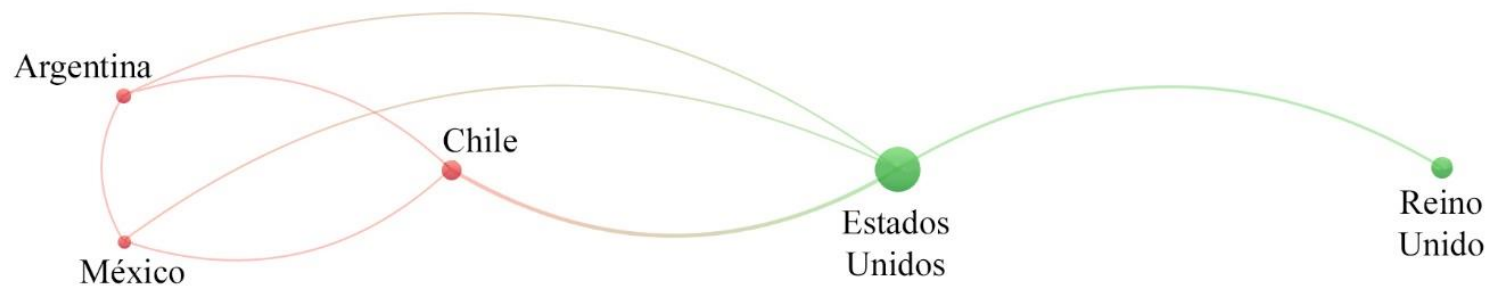

Gráfico 6. Coautorías por país

Nota: Elaboración propia

El top cinco de países más productivos evidencia la presencia de dos clústeres. El clúster 1 (verde) presenta como constructo central a Estados Unidos, el cual dispone de cuatro relaciones de coautorías a través de 44 documentos, la relación con mayor fuerza es la establecida con Reino Unido por medio de 11 documentos producidos. En tanto que, el clúster 2 (rojo) ubica como constructo central a Chile, en este se evidencian tres relaciones a través de 9 artículos, siendo la relación de mayor fuerte la desarrollada con Estados Unidos a través de 4 documentos.

En la productividad por institución, se registra la presencia de 127 centros de afiliación de distinto tipo (universidades, institutos, empresas privadas, instituciones públicas, organizaciones internacionales, etc.); las universidades concentran la mayor cuota de afiliaciones con 77,3\% (99), seguido de los institutos de investigación con el 10,9\% (14).

Como complemento, se construye el ranking de las instituciones más productivas (tabla 6), en el cual liderada la Ohio State University radicada en Estados Unidos que acumula 6 afiliaciones, además de ratificar el liderazgo de los centros universitarios; seguido se ubica The World Bank, cuyas oficinas centrales se ubican en Estados Unidos, con 4 afiliaciones. Dichas instituciones forman parte del constructo central más grande y con mayor concentración de documentos dentro de las relaciones de coautoría por países.

Tabla 6. Instituciones más productivas por autores, autorías y género

\begin{tabular}{|c|l|c|c|c|c|c|}
\hline $\mathbf{R}$ & \multicolumn{1}{|c|}{ Institución } & País & Hombres & Mujeres & A & As \\
\hline 1 & Ohio State University & Estados Unidos & 0 & 6 & 6 & 8 \\
\hline 2 & The World Bank & Estados Unidos & 2 & 2 & 4 & 4 \\
\hline 3 & Indiana University & Estados Unidos & 2 & 2 & 4 & 4 \\
\hline 4 & Griffith University & Australia & 0 & 3 & 3 & 3 \\
\hline 5 & $\begin{array}{l}\text { Universidad Nacional Autónoma de } \\
\text { Nicaragua-León }\end{array}$ & Nicaragua & 3 & 0 & 3 & 3 \\
\hline 6 & Universidad del Desarrollo & Chile & 2 & 1 & 3 & 3 \\
\hline
\end{tabular}




\begin{tabular}{|c|l|c|c|c|c|c|}
\hline 7 & $\begin{array}{l}\text { Universidad Técnica Federico Santa } \\
\text { María }\end{array}$ & Chile & 2 & 1 & 3 & 3 \\
\hline 8 & Colegio de México & México & 0 & 3 & 3 & 3 \\
\hline 9 & Colorado State University & Estados Unidos & 0 & 3 & 3 & 3 \\
\hline 10 & Tulane University & Estados Unidos & 2 & 1 & 3 & 3 \\
\hline 11 & University of California & Estados Unidos & 2 & 1 & 3 & 3 \\
\hline 12 & University of Oregon & Estados Unidos & 0 & 3 & 3 & 3 \\
\hline
\end{tabular}

$* \mathrm{R}=$ Ranking; $\mathrm{A}=$ Autores; As = Autorias.

Nota: Elaboración propia

En relación a las redes de colaboración se pueden establecer de dos tipos: institucional y geográfica, para ello se emplea el porcentaje de artículos con colaboración múltiple los cuales representan el 31,53\% (35) artículos.

De esta porción, el 60\% (21) de los artículos se encuentran elaborados por autores con afiliación de un mismo país, y el 40\% (14) restante son trabajos desarrollados entre autores de procedencias geográficas variadas. En el caso de las colaboraciones nacionales el 57\% (12) son artículos que presentan afiliaciones de autores de distintos centros, y el $43 \%$ (9) son firmados por autores que colaboran en el mismo país y dentro de una misma institución. Con respecto a la colaboración internacional el $100 \%$ de los artículos son firmados por autores procedentes de varias instituciones radicadas en diferentes países.

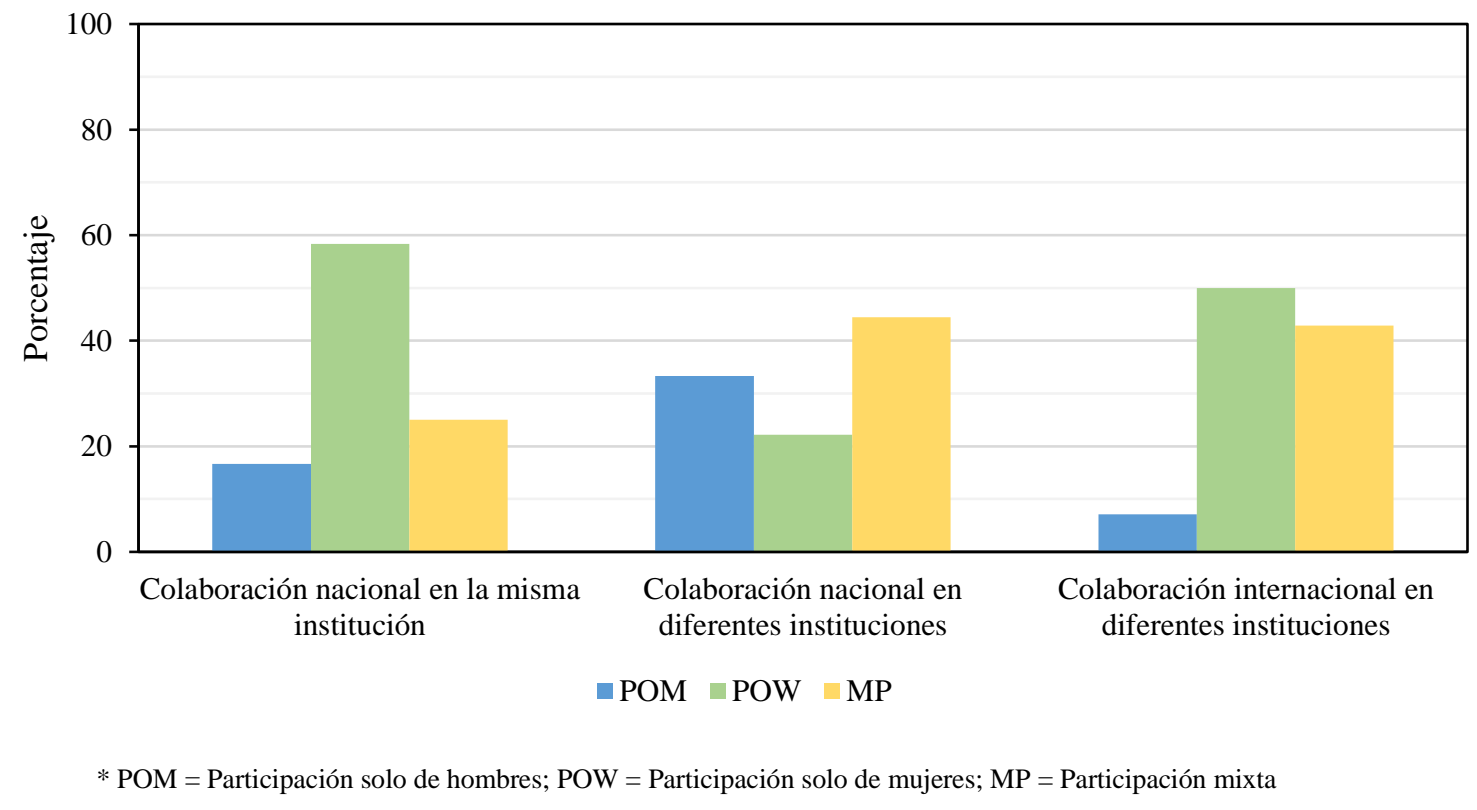

Gráfico 7. Redes de colaboración por género

Nota: Elaboración propia 
En función de las redes de colaboración antes mencionadas, se aprecia que el 58,33\% de trabajos se han efectuado en redes de colaboración nacional de un mismo centro en las cuales únicamente han intervenido mujeres, tendencia que se comparte en las colaboraciones internacionales entre diferentes centros donde la presencia solo de mujeres representa el $50 \%$. Con respecto a las redes de colaboración nacional entre diferentes centros se aprecia que el $44,44 \%$ de los trabajos se han desarrollado bajo la colaboración entre autores de los dos sexos.

\subsection{Revistas}

Para la publicación de los 111 artículos se han utilizado un total de 80 recursos, estableciéndose como la revista más productiva World Development con siete artículos publicados, seguida de Development in Practice con seis publicaciones. Aunque, en relación al total de citas percibidas, lidera Development and Change con 243 citas acumuladas dentro de los estudios publicados, mientras que World Development se ubica en la segunda posición con 195 citas.

Tabla 7. Ranking de las revistas más productivas

\begin{tabular}{|c|l|c|c|c|c|c|c|c|}
\hline $\mathbf{R}$ & \multicolumn{1}{|c|}{ Revista } & País & $\mathbf{f}$ & $\mathbf{f \%}$ & TC & $\begin{array}{c}\text { h- } \\
\text { Index }\end{array}$ & Q & SJR \\
\hline 1 & World Development & Reino Unido & 7 & 6,31 & 195 & 140 & 1 & 2,12 \\
\hline 2 & Development in Practice & Reino Unido & 6 & 5,41 & 8 & 34 & 2 & 0,31 \\
\hline 3 & Gender, Place and Culture & Reino Unido & 5 & 4,50 & 29 & 55 & 1 & 1,10 \\
\hline 4 & Gender and Development & Reino Unido & 3 & 2,70 & 43 & 30 & 1 & 0,64 \\
\hline 5 & Development & Reino Unido & 3 & 2,70 & 3 & 29 & 3 & 0,15 \\
\hline 6 & Latin American Research Review & Estados Unidos & 3 & 2,70 & 77 & 39 & 2 & 0,31 \\
\hline
\end{tabular}

* R = Ranking; $\mathrm{f}=$ Número de artículos publicados; $\mathrm{f} \%$ = Frecuencia relativa; TC = Número de citas percibidas por artículos publicados; h-index = Índice Hirsch; Q = Cuartil; SJR = Índice Scimago Journal Rank.

Nota: Elaboración propia

Sobre la procedencia geográfica de las revistas, éstas se editan principalmente en Reino Unido, proviniendo de allí el 40\% (32), seguido de Estados Unidos con el 33,8\% (27). Además, se aprecia que la distribución por cuartil evidencia que el 50\% de las revistas se encuentran ubicadas en el primer cuartil del Índice SJR (gráfico 8). 


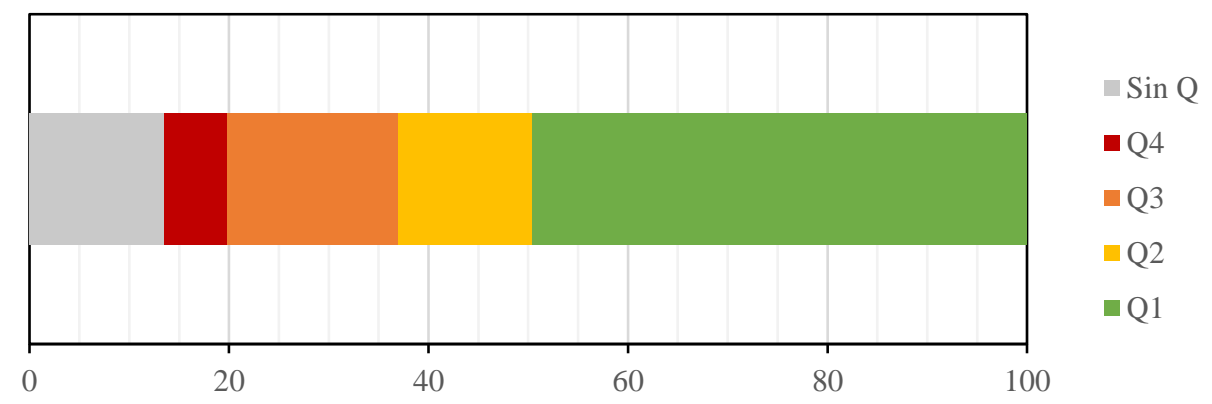

Gráfico 8. Distribución de la revistas según cuartil

Nota: Elaboración propia

Adicional a ello, se ha calculado el Índice de Dispersión, que es de 1,39 artículos/revistas, en un contexto en el que el $80 \%$ de las revistas solo han publicado un artículo sobre esta temática. En complemento, dentro del análisis de recursos se puede identificar los núcleos de concentración generados en relación a la producción científica de esta temática; para ello se aplica la Ley de Bradford (1934), misma que propone que, al examinar la producción de cualquier campo científico, es posible identificar un elevado porcentaje de estudios concentrados en un reducido número de revistas.

Para conseguir comprobar dicha ley, es necesario establecer la zona mínima de Bradford (MBZ) la cual toma un valor de 32; de esta manera al ordenar de forma descendente el ranking de revistas en función a su productividad, se puede determinar que el núcleo de Bradford, corresponde al grupo de revistas que sumada su productividad sea igual a 32. Con lo antes establecido, se aprecia que el núcleo de Bradford se integra por 33 revistas tal como se puede evidenciar dentro del gráfico 9.

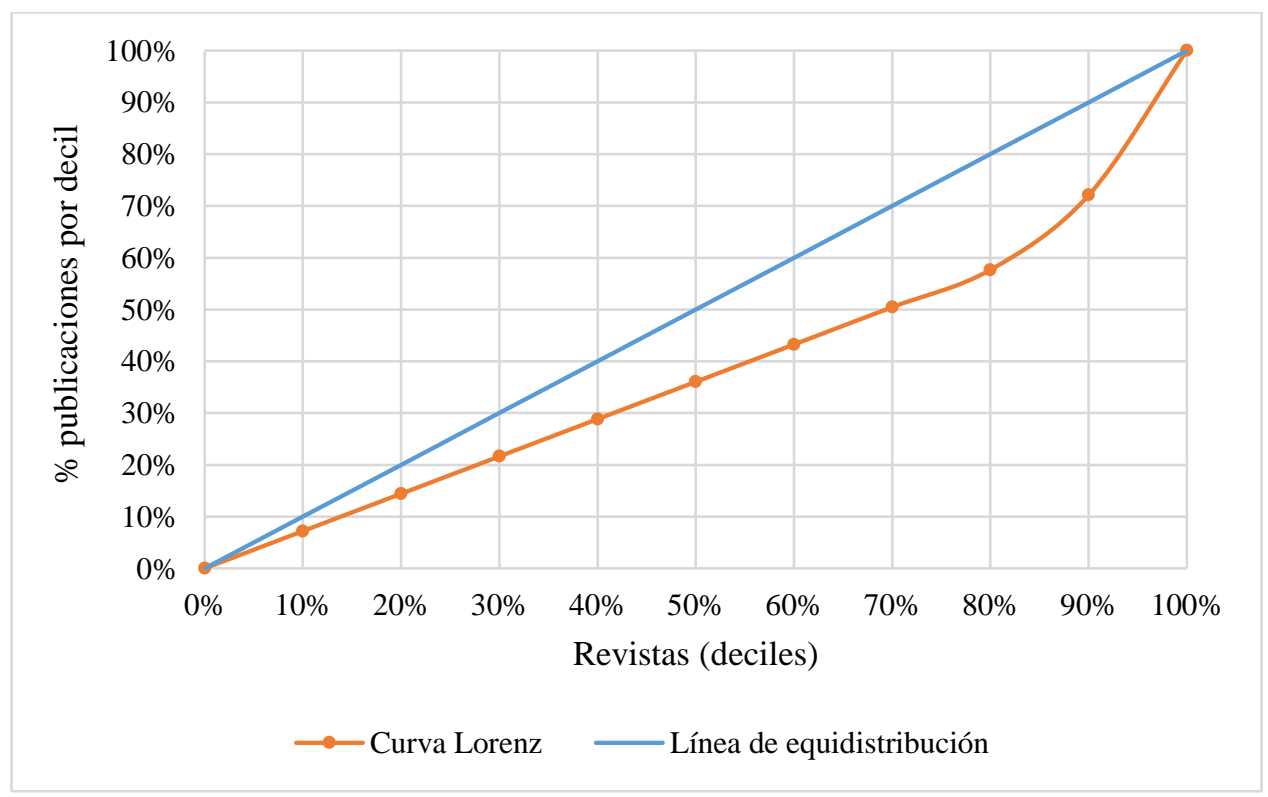

Gráfico 9. Curva de Lorenz - Núcleo de Bradford de las revistas más productivas

Nota: Elaboración propia 


\section{6. Áreas temáticas}

A continuación, se analizan las áreas temáticas a través de las cuales se encuentran clasificados los recursos dentro de Scopus. En relación al área de conocimiento, el predominio lo establece Ciencias Sociales con 71 artículos (64\%) y un total de 964 citas acumuladas, seguida de Artes y Humanidades con 13 artículos $(11,7 \%)$ y 108 citas.

Tabla 8. Clasificación de los artículos por área temática

\begin{tabular}{|c|l|c|c|c|c|}
\hline $\mathbf{R}$ & \multicolumn{1}{|c|}{ Área } & J & $\mathbf{f}$ & TC & C/f \\
\hline 1 & Ciencias Sociales & 47 & 71 & 964 & 13,6 \\
\hline 2 & Artes y Humanidades & 9 & 13 & 108 & 8,3 \\
\hline 3 & Negocios, Gestión y Contabilidad & 6 & 6 & 25 & 4,2 \\
\hline 4 & Economía, Econometría y Finanzas & 5 & 6 & 67 & 11,2 \\
\hline 5 & Medicina & 4 & 4 & 10 & 2,5 \\
\hline 6 & Ciencia medioambiental & 2 & 3 & 63 & 21,0 \\
\hline 7 & Bioquímica, Genética y Biología Molecular & 2 & 2 & 38 & 19,0 \\
\hline 8 & Ciencias de la Tierra y Planetarias & 2 & 2 & 1 & 0,5 \\
\hline 9 & Profesiones de la salud & 1 & 2 & 11 & 5,5 \\
\hline 10 & Sin información & 2 & 2 & 43 & 21,5 \\
\hline
\end{tabular}

* R = Ranking; J = revistas; $\mathrm{f}=$ número de artículos publicados; $\mathrm{TC}$ = número de citas percibidas por artículos publicados; $\mathrm{C} / \mathrm{f}=$ promedio de citas recibidas por artículos publicados.

Nota: Elaboración propia

En relación a las categorías, se destaca como líder la categoría de Desarrollo con 34 artículos en 17 revistas, seguido de Geografía, Planificación y Desarrollo, con 16 artículos en 11 revistas. La categoría con mayor número de citas es Desarrollo con 582 citas.

\subsection{Palabras claves}

El uso correcto de las palabras claves dentro los estudios se puede convertir en una tarea compleja de alcanzar y que en mucho de los casos es poco reconocida. Se puede apreciar que los autores tienden a utilizar términos sencillos que les permitan alcanzar la mayor coincidencia de sus estudios en las búsquedas generales que se desarrollan en la comunidad científica, pero desafortunadamente esto no asegura que el término exprese en su totalidad el objetivo alcanzado por el documento, razón por la cual es necesario resaltar que para la obtención de resultados de calidad dentro de las búsquedas avanzadas es necesario desarrollar un mayor esfuerzo para que estos términos se conviertan en descriptores correctos de la investigación (Álvarez-García et al., 2019).

Las palabras claves en los últimos años se constituyen como el mecanismo de mayor uso para la identificación de documentos por parte de la comunidad científica. A pesar de la relevancia que estas presentan, hasta la actualidad es posible observar artículos que carecen 
de palabras claves, por una parte, debido al formato de divulgación el cual no incluye este criterio, factor que provoca su inadecuada ubicación dentro de los metadatos de las distintas bases. En el presente estudio se reporta que el $46 \%$ (51) de los documentos no disponen de esta información.

Con los 60 documentos restantes, se estableció la presencia de 230 palabras claves empleadas en conjunto 354 veces, a continuación, dentro de la tabla 9 se presentan las palabras claves con el mayor número de apariciones. El término género se establece como el descriptor central al obtener 24 apariciones dentro de los 111 documentos de estudio, seguida de América Latina con el mismo número de repeticiones.

Tabla 9. Distribución de la palabras claves

\begin{tabular}{|c|l|c|c|l|c|}
\hline R & \multicolumn{1}{|c|}{ Palabras claves } & f & R & \multicolumn{1}{c|}{ Palabras claves } & f \\
\hline 1 & Gender & 24 & 29 & Entrepreneurship & 3 \\
\hline 2 & Latin America & 24 & 30 & Gender and diversity & 3 \\
\hline 3 & Men & 9 & 31 & Gender inequality & 3 \\
\hline 4 & Working women & 7 & 32 & Globalization & 3 \\
\hline 5 & Gender roles & 6 & 33 & Informality & 3 \\
\hline 6 & Economic development & 5 & 34 & Labour and livelihoods & 3 \\
\hline 7 & Ecuador & 5 & 35 & $\begin{array}{l}\text { Latin America and the } \\
\text { Caribbean }\end{array}$ & 3 \\
\hline 8 & gender equality & 5 & 36 & Mexico & 3 \\
\hline 9 & poverty & 5 & 37 & Neoliberalism & 3 \\
\hline 10 & Employment & 4 & 38 & Women's rights & 3 \\
\hline 11 & Women & 4 & & & \\
\hline
\end{tabular}

$* \mathrm{R}=$ Ranking; $\mathrm{f}=$ frecuencia.

Nota: Elaboración propia

\section{Conclusiones}

Este tipo de estudios constituyen en los últimos años una herramienta de consulta de gran valor para los nuevos investigadores, debido a que esta presenta un panorama detallado de la evolución y estado actual que la temática de estudio presenta. De esta manera, se pone a disposición de los interesados información como: autores más productivos en función de citas y documentos producidos, principales revistas interesadas en la publicación de temas relacionados con la temática, países e instituciones centradas en el desarrollo de investigaciones relacionadas, entre otros.

Luego de la aplicación de todos los indicadores antes mencionados, el análisis bibliométrico revela que la publicación de artículos científicos que tratan explícitamente sobre género y economía en Latinoamérica, y que están indexados en Scopus, se inicia en 1985. Ello coincide con la afirmación previamente realizada por Caulfield (2001), que ubica a mediados de la década de 1980 como el punto de surgimiento considerable de artículos científicos que ubican al género como categoría central de análisis en la región.

En la actualidad, se dispone de 34 años de literatura científica consolidados en 111 artículos que fundamentan la temática. El año de mayor producción es 1998; sin embargo, este año no 
se desarrollan redes de colaboración notables, pues de los 13 documentos publicados ese año, 8 de ellos se desarrollaron de forma individual. Por su parte, el año con mayor número de citas es 2002 con 258 citas.

La línea de evolución de la temática determina que, por la distribución de la misma, ésta se encuentra en un crecimiento exponencial que corresponde a la segunda fase de comportamiento de la producción que indica López López (1996), con lo cual esta temática se encuentra lejos de alcanzar un crecimiento de ajuste lineal, lo que evidencia que la temática aún es muy joven en su estudio, principalmente por la presencia de un mayor número de estudios cualitativos-exploratorios.

En relación a la producción por autores, se aprecia un predominio de investigadores transitorios. Por el contrario, la ausencia de grandes productores ratifica la juventud de la temática. Por otra parte, en función del crecimiento que presenta la temática se evidencia el surgimiento de un reducido grupo de autores aspirantes que presenta entre 2 a 3 estudios, aunque el $90 \%$ de estos registran como afiliación geográfica en potencias para el desarrollo de investigación a nivel internacional como Estados Unidos y Reino Unido, y tan solo un autor del grupo de aspirantes es procedente de la zona de estudio al registrar su afiliación en Uruguay. Este grupo representa el 3\% del total de autores y concentra el 10\% de los artículos.

En lo que se refiere al sexo de los autores, se evidencia una mayor presencia de mujeres con el $69,8 \%$ frente a un 30,2\% de hombres; dicha distribución que se aprecia claramente dentro de las dos fases de comportamiento registradas en la línea de evolución, en las cuales existe un predominio de la mujer, aunque paulatinamente se aprecia la incorporación del hombre en estos estudios. En relación a las citas percibidas por la producción de estudio, los tres documentos que acumulan la mayor cantidad de citas presentan una participación equitativa ( 2 mujeres y 2 hombres), aunque el documento con el mayor promedio de citas/años es el trabajo de Molyneux (2002), el cual corresponde a una autora femenina de gran relevancia. Así también, se identifica que el 100\% de los autores que integran el ranking de los más productivos son mujeres.

Las tendencias de colaboración fue otro espacio en el que se evidenció el predominio de la mujer, al producir el $85,5 \%$ de los documentos sin colaboración y el 52,4\% de los documentos elaborados por pares (tendencia de colaboración con mayor preferencia); en tanto que, los documentos producidos entre tres autores evidencian una producción del $40 \%$ tanto por grupos solo integrados por mujeres, así como por grupos de participación mixta, aunque en la producción de documentos de cuatro autores o más predomina una participación mixta en la elaboración de los mismos. Ello encaja con las afirmaciones de Söderlund \& Madison (2015) y Caro González \& Guarinos (2017) sobre la tendencia a una mayor presencia femenina en la investigación sobre temas relacionados al género en una amplia gama de disciplinas.

En relación a las afiliaciones se aprecia una amplia variedad de latitudes con respecto a la afiliación tanto geográfica como institucional. Con respecto a la afiliación institucional, son las universidades las que se establecen como el centro de mayor preferencia para las afiliaciones con el 77,3\%, siendo la Ohio State University radicada en Estados Unidos, la universidad con mayor número de afiliaciones.

En tanto que, la afiliación geográfica revela que Estados Unidos es el líder al concentrar 72 autores, 76 autorías y 50 centros. Con respecto a la tendencia de coautoría entre los países, 
se aprecia una red de colaboración de dos constructos en las cuales el nodo principal es Estados Unidos, siendo la relación más fuerte de toda la red la que mantiene con Reino Unido en el clúster 1, dando cuenta que la colaboración y comunicación entre los autores y centros radicados en estos dos países es mucho más estrecha que con Chile, Argentina y México. Adicional a ello, de forma general se observa que la producción de trabajos se desarrolla en su mayoría en una combinación de dos autores con un Índice de Colaboración de 1,59 autores/articulo, tendencia que está contribuyendo a la consolidación de estudios de mayor impacto al aumentar la participación de autores.

Lo antes señalado confirma la observación de Wöhrer (2016) y Tsay \& Li (2017) acerca de que la mayoría de la investigación en torno a temas de género proviene de los centros occidentales tradicionales del conocimiento; lo que en este caso se mantiene, pese a que el foco central de investigación es una región del Sur global.

Con respecto a la dispersión para la publicación de los documentos, se evidencia que no existen núcleos de concentración para la publicación de la información en relación a la temática de estudio, debido a que, el núcleo de Bradford es igual a 32, estando este concentrado en 33 revistas, dicha relación que evidencia una alta dispersión de la información, lo cual se ratifica el identificar 64 revistas con un solo artículo publicado.

Las revistas con mayor concentración de artículos son: World Development y Development in Practice, las cuales reúnen el 12\% de los artículos y un total de 203 citas, a pesar de ello es Development and Change con 243 citas acumuladas quien es la revista más citada.

Finalmente, el área de clasificación predominante en las revistas identificadas es Ciencias Sociales con el 59\% de las revistas. Así también, se observa que el 50\% del total de los recursos se encuentran indexado en el primer cuartil de sus respectivas áreas, mientras que el 14\% de los recursos carecen del cálculo del cuartil por un lado por ser de reciente reingreso o porque han sido apartadas de su indexación.

\section{Limitaciones}

Este estudio ha pretendido plasmar de forma descriptiva y cuantitativa cómo se encuentra y cómo ha evolucionado el estudio de género y economía en Latinoamérica, descartando la intención de valorar la calidad de los artículos, lo cual no deja de contemplarse como alternativa de planteamiento para estudios posteriores, debido a la necesidad de ampliar la investigación hacia otros niveles.

En ese contexto, debe aclararse que dentro del presente estudio se consideraron solo términos en inglés en una región de habla hispana y portuguesa, debido a que la mayoría de los documentos publicados en revistas científicas de alto impacto - objeto de estudio del presente documento - se elaboran en dicho idioma o cuentan, al menos, con títulos, resúmenes y palabras claves traducidos también al inglés; sin embargo, reconocemos que existe la posibilidad de que no todos los artículos en español o portugués en el área presenten una traducción de sus títulos y palabras claves al inglés, lo que puede llevar a subestimar la cantidad de producción científica que se identificó dentro de la temática.

Además de ello, existe también una cantidad considerable de investigación científica indexada en bases regionales o de menor impacto que no han sido consideradas por los 
motivos antes expuestos, pero cuyo estudio futuro podría incrementar la comprensión de esta temática. Por su parte, el uso de la base de datos Scopus como fuente de aproximación a las revistas de mayor impacto se planteó bajo dos criterios, por un lado, se ha evidenciado un aumento en las suscripciones a la misma por parte del mundo académico de América Latina, en comparación a WoS que es de acceso mucho más restringido para los investigadores; mientras que, por otro, esta base de datos brinda acceso a metadatos de mejor calidad, elemento que permite un análisis más adecuado de los documentos que se identificaron.

Para finalizar, debe destacarse que, dado el propósito de la presente investigación, la búsqueda se centró estudios que considerasen al género como una categoría de análisis, ampliando así las posibilidades de identificación de estudios dentro de la ecuación de búsqueda. No obstante, debe señalarse que el uso del término mujeres pudo haber sido relevante a la temática también, sobre todo al enfocarse en estudios previos a mediados de la década de 1980.

\section{Lista de referencias}

Abbassi, J., \& Lutjens, S. (2002). Rereading Women in Latin America and the Caribbean. The Political Economy of Gender. Lanham, MD: Rowman \& Littlefield.

Abramo, L. (1998). The sociology of work in Latin America: A complex development and current challenges. Work and Occupations, 25(3), 305-332. doi: 10.1177/0730888498025003003

Alonso, S., Cabrerizo, F. J., Herrera-Viedma, E., \& Herrera, F. (2009). H-Index: A review focused in its variants, computation and standardization for different scientific fields. Journal of Informetrics, 3(4), 273-289. doi: 10.1016/j.joi.2009.04.001

Álvarez-García, J., Durán-Sánchez, A., \& del Río-Rama, M. C. (2018). Scientific Coverage in Community-Based Tourism: Sustainable Tourism and Strategy for Social Development. Sustainability, 10(4), 1158. doi: 10.3390/su10041158

Álvarez-García, J., Maldonado-Erazo, C. P., Del Río-Rama, D. M., \& Castellano-Álvarez, J. F. (2019). Cultural Heritage and Tourism Basis for Regional Development: Mapping of Scientific Coverage. Sustainability, 11(21). doi: 10.3390/su11216034

Andrés, A. (2009). Measuring academic research: How to undertake a bibliometric study: Elsevier.

Aslanbeigui, N., Pressman, S., \& Summerfield, G. (1994). Women in the age of economic transformation: gender impact of reforms in post-socialist and developing countries London: Routledge.

Benería, L., \& Roldán, M. (1992). Las encrucijadas de clase y género. México: Fondo de Cultura Económica.

Blau, F., Ferber, M., \& Winkler, A. (2002). The Economics of Women, Men and Work. Upper Saddle River: Prentice Hall.

Blay, E. A. (1978). Trabalho domesticado: a mulher na indústria paulista. Sao Paulo: Editora Átiica. 
Bonilla, C. A., Merigó, J. M., \& Torres-Abad, C. (2015). Economics in Latin America: A bibliometric analysis. Scientometrics, 105(2), 1239-1252. doi: 10.1007/s11192-0151747-7

Bradford, S. C. (1934). Sources of information on specific subjects. Engineering, 137, 8586.

Brockman, C. T. (1985). Women and development in northern Belize. Journal of Developing Areas 19(4), 501-514.

Cabral, E. (1994). No going back. Mexican women find opportunity and obstacles in a changing economy. Ford Foundation report (New York, N.Y. : 1992), 25(3), 11-17.

Caro, F. J., \& Guarinos, V. (2017). Male presence in gender research networks in the communication field in Spain. Masculinities and Social Change, 6(1), 62-90. doi: 10.17583/MCS.2017.2452

Carrasco, C. (2003). Tiempos, trabajos y organización social: reflexiones en torno al mercado laboral femenino, Mujeres y economía. Barcelona: Icaria.

Castaño, C. (1999). Economía y género. Política y sociedad, 32, 23-42.

Caulfield, S. (2001). The history of gender in the historiography of Latin America. The Hispanic American Historical Review, 81(3), 449-490. doi: 10.1215/00182168-81-34-449

Cislak, A., Formanowicz, M., \& Saguy, T. (2018). Bias against research on gender bias. Scientometrics, 115(1), 189-200. doi: 10.1007/s11192-018-2667-0

Crane, D. (1977). Social structure in a group of scientists: a test of the "invisible college" hypothesis. In S. Leinhardt (Ed.), Social Networks (pp. 161-178): Academic Press.

Cravey, A. J. (1997). The politics of reproduction: Households in the Mexican industrial transition. Economic Geography, 73(2), 166-186

Cvencek, D., Meltzoff, A. N., \& Greenwald, A. G. (2011). Math-gender stereotypes in elementary school children. Child Dev, 82(3), 766-779. doi: 10.1111/j.14678624.2010.01529.x

De Barbieri, T. (2004). Más de tres décadas de los estudios de género en América Latina. Revista Mexicana de Sociología, 66, 197-214. doi: 10.2307/3541450

de Lattes, Z. R., Sautu, R. A., \& Wainerman, C. H. (1977). Participación de las mujeres en la actividad econ6mica de la Argentina, Bolivia y Paraguay: El caso argentino (Vol. 1-2). Buenos Aires: CENEP.

Deere, C. D., \& León, M. (2000). Género, propiedad y empoderamiento: Tierra, Estado y mercado en América Latina. Bogotá: Universidad Nacional de Colombia.

Desposato, S., \& Norrander, B. (2009). The Gender Gap in Latin America: Contextual and individual influences on gender and political participation. British Journal of Political Science, 39(1), 141-162. doi: 10.1017/S0007123408000458

Durán-Sánchez, A., Álvarez-García, J., Del Río-Rama, M. C., \& Sarango-Lalangui, P. (2018). Analysis of the Scientific Literature Published on Smart Learning. ESPACIOS, 39(10), 7-24.

Ellemers, N. (2018). Gender Stereotypes. Annual Review of Psychology, 69, 275-298. doi: https://doi.org/10.1146/annurev-psych-122216-011719

Ellis, M., Conway, D., \& Bailey, A. J. (1996). The circular migration of Puerto Rican women: Towards a gendered explanation. International Migration, 34(1), 31-58

Escorcia-Otálora, T. A., \& Poutou-Piñales, R. A. (2008). Análisis bibliométrico de los artículos originales publicados en la revista Universitas Scientiarum (1987-2007). Universitas Scientiarum, 13(3), 236-244. 
Esquivel, V. (2011). La economía del cuidado en América Latina. Panamá: PNUD. European Commision. (2019). She figures 2018. . Brussels: European Union.

Frank, M. (2006). Access to the Scientific Literature - A Difficult Balance. New England Journal of Medicine, 354(15), 1552-1555. doi: 10.1056/NEJMp068004

Fuwa, N. (2000). The poverty and heterogeneity among female-headed households revisited: The case of Panama. World Development, 28(8), 1515-1542. doi: 10.1016/S0305750X $(00) 00036-X$

Georges, E. (1992). Gender, Class, and Migration in the Dominican Republic: Women's Experiences in a Transnational Community: GENDER, CLASS, AND MIGRATION. Annals of the New York Academy of Sciences $\quad 645(1), \quad$ 81-99. doi: 10.1111/j.1749-6632.1992.tb33487.x

Goldschmidt, P. G. (1986). Information synthesis: a practical guide. Health Services Research, 21(2 Pt 1), 215-237.

Handley, I. M., Brown, E. R., Moss-Racusin, C. A., \& Smith, J. L. (2015). Quality of evidence revealing subtle gender biases in science is in the eye of the beholder. Proceedings of the National Academy of Sciences, 112(43), 13201-13206. doi: https://doi.org/10.1073/pnas.1510649112

Hays-Mitchell, M. (1995). Voices and visions from the streets: gender interests and political participation among women informal traders in Latin America. Environment \& Planning D: Society \& Space, 13(4), 445-469. doi: 10.1068/d130445

Hays-Mitchell, M. (1997). Development vs empowerment: the gendered legacy of economic restructuring in Latin America. Yearbook - Conference of Latin Americanist Geographers, 23, 119-131.

Hays-Mitchell, M. (2002). Resisting austerity: A gendered perspective on neo-liberal restructing in Peru. Gender and Development, 10(3), 71-81. doi: $10.1080 / 13552070215920$

Hernández-González, V., Sans-Rosell, N., Jové-Deltell, M. C., \& Reverter-Masia, J. (2016). Comparación entre Web of Science y Scopus, Estudio Bibliométrico de las Revistas de Anatomía y Morfología. Int. J. Morphol., 34(4), 1369-1377.

Hill, D., Larcombe, I., \& Refshauge, J. G. (1978). Smoking and impairment of performance. Medical Journal of Australia, 2(2), 60-63.

Hite, A. B., \& Viterna, J. S. (2005). Gendering class in Latin America: How women effect and experience change in the class structure. Latin American Research Review, 40(2), $50-82$

Holman, L., Stuart-Fox, D., \& Hauser, C. E. (2018). The gender gap in science: How long until women are equally represented? PLoS Biology, 16(4). doi: https://doi.org/10.1371/journal.pbio.2004956

Howes, C., \& Singh, A. (1995). Long-term trends in the World economy: The gender dimension. World Development, 23(11), 1895-1911. doi: 10.1016/0305750X(95)00096-U

Hubert, J. J. (1981). General bibliometric models. Ontario: Libr Trends.

Jelin, E. (1974). Formas de organización de la actividad económica y estructura ocupacional: El caso de Salvador, Brasil. Desarrollo Económico, 14(53), 181-203. doi: $10.2307 / 3466052$

Kidder, T. (1999). Alternative financial institutions? Sustainability, development, social reproduction, and gender analysis. Development in practice, 9(4), 482-487. 
Laurie, N. (1997). From work to welfare: The response of the Peruvian state to the feminization of emergency work. Political Geography, 16(8), 691-714. doi: 10.1016/S0962-6298(96)00078-9

Laurie, N. (1999). State-backed work programmes and the regendering of work in Peru: Negotiating femininity in 'the provinces'. Environment and Planning A, 31(2), 229250. doi: 10.1068/a310229

León de Leal, M. (1977). Mujer y el desarrollo en Colombia. Bogota: ACEP.

López López, P. (1996). Introducción a la bibliometría. Valencia: Promolibro.

Lovell, P. A. (2006). Race, gender, and work in São Paulo, Brazil, 1960-2000. Latin American Research Review, 41(3), 63-87.

Lustig, N., Rendon, T., \& Bunster, X. (1979). Female Employment, Occupational Status, and Socioeconomic Characteristics of the Family in Mexico. Signs: Journal of Women in Culture and Society, 5(1), 143-153. doi: 10.1086/493690

Machado Neto, Z. (1978). As meninas: Sobre o trabalho da crianca e da adolescente na familia de populac6es faveladas. Paper presented at the Seminar on Women in the Labor Force in Latin America, Rio de Janeiro.

Madeira, F. R. (1978). El trabajo de la mujer en fortaleza. Demografía y economía, 12(1), 46-74.

Merigó, J. M., Mas-Tur, A., Roig-Tierno, N., \& Ribeiro-Soriano, D. (2015). A bibliometric overview of the Journal of Business Research between 1973 and 2014. Journal of Business Research, 68(12), 2645-2653. doi: 10.1016/j.jbusres.2015.04.006

Molinas, J. R. (1998). The impact of inequality, gender, external assistance and social capital on local-level cooperation. World Development, 26(3), 413-431. doi: 10.1016/S0305750X(97)10066-3

Molyneux, M. (2002). Gender and the silences of social capital: Lessons from Latin America. Development and Change, 33(2), 167-188. doi: 10.1111/1467-7660.00246

Morales i Perez, S. (1999). Agribusiness, rural development and gender in Latin America: Work and daily life of working women in Santa Rosa (Mendoza, Argentina). Documents d'Analisi Geografica(35), 121-145.

Moreno, C. (1996). An experience of women's participation in strengthening community management. Habitat debate / UNCHS (Habitat), the United Nations Centre for Human Settlements, 2(1), 11.

Morgan, J., \& Buice, M. (2013). Latin American attitudes toward women in politics: The influence of elite cues, female advancement, and individual characteristics. American Political Science Review, 107(4), 644-662. doi: 10.1017/S0003055413000385

Nash, J., \& Safa, H. (1976). Sex and Class in Latin America. New York: Praeger Publishers.

Navarro, M. (1979). Research on Latin American Women. Signs: Journal of Women in Culture and Society, 5(1), 111-120.

Pacheco, E., \& Blanco, M. (1998). Three modes of analysis in the incorporation of a gender perspective in socio-demographic studies of urban labor in Mexico. Papeles de población / Centro de Investigación y Estudios Avanzados de la Población, Universidad Autónoma del Estado de México, 4(15), 73-94.

Pescatello, A. (1972). The female in Ibero-America: an essay on research bibliography and research directions. Latin American Research Review, 7(2), 125-141.

Podsakoff, P. M., MacKenzie, S. B., Podsakoff, N. P., \& Bachrach, D. G. (2008). Scholarly influence in the field of management: A bibliometric analysis of the determinants of 
university and author impact in the management literature in the past quarter century. Journal of Management, 34(4), 641-720. doi: 10.1177/0149206308319533

Price, D. J. (1956). The exponential curve of science. Discovery, 17(6), 240-243.

Radcliffe, S. A. (1990). Between hearth and labor market: the recruitment of peasant women in the Andes. International Migration Review, 24(2), 229-249. doi: 10.2307/2546550

Raynolds, L. T. (1998). Harnessing women's work: restructuring agricultural and industrial labor forces in the Dominican Republic. Economic Geography, 74(2), 149-169

Reyes, G. (2011). Centros urbanos de América Latina 1997, 2006: Disparidades salariales según género y crecimiento económico Revista de Ciencias Sociales, 131-132. doi: 10.15517/rcs.v0i131-132.3899

Rico, M. N. (1998). Women in water-related processes in Latin America: Current situation and research and policy proposals. International Journal of Water Resources Development, 14(4), 461-471. doi: 10.1080/07900629849097

Roberts, J. T., \& Dodoo, F. N. A. (1995). Population growth, sex ratios, and women's work on the contemporary Amazon frontier. Yearbook - Conference of Latin Americanist Geographers, 21, 91-105.

Rodriguez Enriquez, C. (2015). Economía feminista y economía del cuidado: Aportes conceptuales para el estudio de la desigualdad. Nueva Sociedad, 256(3), 1-15.

Rodríguez Enríquez, C. (2007). Economía del cuidado, equidad de género y nuevo orden económico internacional. In A. Giron \& E. Correa (Eds.), Del Sur hacia el Norte: Economía política del orden económico internacional emergente. Buenos Aires: CLACSO, Consejo Latinoamericano deCiencias Sociales.

Rowlands, J. (1992). Women, empowerment and development in Honduras. Graduate Discussion Paper - University of Durham, Department of Geography, 92-2.

Saffioti, H. (1969). Women in Class Society. New York: Monthly Review Press.

Salvador, S. (2007). Comercio, género y equidad en América Latina: Generando conocimiento para la Acción Política: Estudio comparativo de la "economía del cuidado” en Argentina, Brasil, Chile, Colombia, México y Uruguay. Uruguay: Red Internacional de Género y Comercio, Capítulo Latinoamericano.

Schmukler, B. (1978). La mujer en empresas familiares como trabajadora familiar no remunerada y trabajadora por cuenta propia dentro del sector comercio de alimentos en Argentina. Paper presented at the Seminar on Women in the Labor Force in Latin America, Rio de Janeiro.

Shen, H. (2013). Inequality quantified: Mind the gender gap. Nature News(495), 22-24. doi: $10.1038 / 495022 \mathrm{a}$

Söderlund, T., \& Madison, G. (2015). Characteristics of gender studies publications: a bibliometric analysis based on a Swedish population database. Scientometrics, 105(3), 1347-1387. doi: 10.1007/s11192-015-1702-7

Spinak, E. (1996). Diccionario enciclopédico de bibliometría, cienciometría e informetría. Caracas: UNESCO CII/II.

Steinke, J., Lapinski, M. K., Crocker, N., Zietsman-Thomas, A., Williams, Y., Evergreen, S., \& Kuchibhotla, S. (2007). Assessing media influences on middle school aged children's perceptions of women in science using the Draw-A-Scientist Test (DAST). Science Communication, 29(1), 35-64. doi: 10.1177/1075547007306508

Stephen, L. (2005). Women's weaving cooperatives in Oaxaca: An indigenous response to neoliberalism. Critique of Anthropology, 25(3), 253-278. doi: 10.1177/0308275X05055215 
Teele, D. L., \& Thelen, K. (2017). Gender in the Journals: Publication Patterns in Political Science. PS - Political Science and Politics, 50(2), 433-447. doi: https://doi.org/10.1017/S1049096516002985

Teissedre, S. (1997). First Mayan Women's Congress. UN chronicle, 34(4), 57.

Terjesen, S., \& Amorós, J. E. (2010). Female entrepreneurship in Latin America and the caribbean: Characteristics, drivers and relationship to economic development. European Journal of Development Research, 22(3), 313-330. doi: 10.1057/ejdr.2010.13

Tsay, M. Y., \& Li, C. N. (2017). Bibliometric analysis of the journal literature on women's studies. Scientometrics, 113(2), 705-734. doi: 10.1007/s11192-017-2493-9

Van der Lee, R., \& Ellemers, N. (2018). Perceptions of gender inequality in academia: Reluctance to let go of individual merit ideology. In B. T. Rutjens \& M. J. Brandt (Eds.), Belief Systems and the Perception of Reality (pp. 63-78): Taylor and Francis Inc.

Vásconez, A. (2017). Crecimiento económico y desigualdad de género: análisis de panel para cinco países de América Latina. Revista CEPAL(122), 85-113.

Wiig, H. (2013). Joint titling in rural peru: Impact on women's participation in household decision-making. World Development, 52, 104-119. doi: 10.1016/j.worlddev.2013.06.005

Wöhrer, V. (2016). Gender studies as a multi-centred field? Centres and peripheries in academic gender research. Feminist Theory, 17(3), 323-343. doi: $10.1177 / 1464700116652840$ 